\title{
On the Links-Gould Invariant of Links
}

\author{
David De Wit* Louis H Kauffman ${ }^{\dagger}$ and Jon R Links*
}

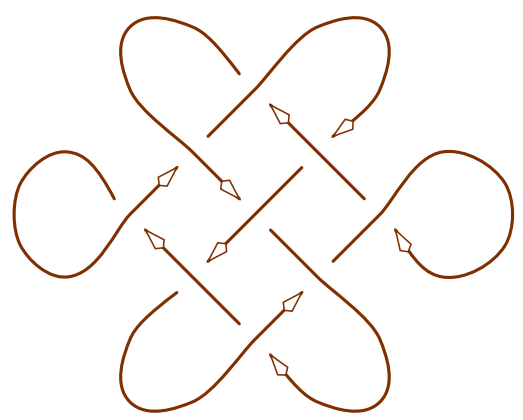

\begin{abstract}
We introduce and study in detail an invariant of $(1,1)$ tangles. This invariant, derived from a family of four dimensional representations of the quantum superalgebra $U_{q}[g l(2 \mid 1)]$, will be referred to as the Links-Gould invariant. We find that our invariant is distinct from the Jones, HOMFLY and Kauffman polynomials (detecting chirality of some links where these invariants fail), and that it does not distinguish mutants or inverses. The method of evaluation is based on an abstract tensor state model for the invariant that is quite useful for computation as well as theoretical exploration.
\end{abstract}

\section{Introduction}

Since the discovery of the Jones polynomial [14], several new invariants of knots, links and tangles have become available due to the development of sophisticated mathematical techniques. Among these, the quantum algebras as defined by Drinfeld [9] and Jimbo [13], being examples of quasi-triangular Hopf algebras, provide a systematic means of solving the Yang-Baxter equation and in turn may be employed to construct representations of the braid group. From each of these representations, a prescription exists to compute invariants of oriented knots and links [34, 39, 41], from which the Jones polynomial is recoverable using the simplest quantum algebra $U_{q}[s l(2)]$ in its minimal (2-dimensional) representation.

* David De Wit and Jon R Links: Department of Mathematics, The University of Queensland. Q, 4072, Australia. email: ddw@maths.uq.edu.au, jrl@maths.uq.edu.au.

${ }^{\dagger}$ Louis H Kauffman: Department of Mathematics, Statistics and Computer Science, The University of Illinois at Chicago. 851 South Morgan Street, Chicago IL, 60607-7045, USA. email: kauffman@uic.edu. 
From such a large class of available invariants, it is natural to ask if generalisations exist, with the view to gaining a classification. One possibility is to look to multiparametric extensions in order to see which invariants occur as special cases. A notable example is the HOMFLY invariant [10] which includes both the Jones and Alexander-Conway invariants 2, 6] as particular cases as well as the invariants arising from minimal representations of $U_{q}[s l(n)]$ [39]. Another is the Kauffman polynomial which includes the Jones invariant as well as those obtained from the quantum algebras $U_{q}[o(n)]$ and $U_{q}[s p(2 n)]$ in the $q$-deformations of the defining representations [39].

The work of Turaev and Reshetikhin 35] shows that the algebraic properties of quantum algebras are such that an extension of this method to produce invariants of oriented tangles is permissible. A tangle diagram is a link diagram with free ends. An associated invariant takes the form of a tensor operator acting on a product of vector spaces. Zhang 40] has extended this formalism to the case of quantum superalgebras which are $\mathbb{Z}_{2}$-graded generalisations of quantum algebras.

Since quantum superalgebras give rise to nontrivial one-parameter families of irreducible representations, it is possible to utilise them for the construction of two variable invariants. This was first shown by Links and Gould [26] for the simplest case using the family of four dimensional representations of $U_{q}[g l(2 \mid 1)]$. It was also made known that a one variable reduction of this invariant coincides with a one variable reduction of the Kauffman polynomial by the use of the Birman-Wenzl-Murakami algebra. Extensions to more general representations of quantum superalgebras are discussed in [12].

Thus far, little has been investigated with regard to the Links-Gould invariant. Here we report on some properties and behaviour. The method of evaluating the invariant involves a prior construction of the quantum $R$-matrix associated with a family of four dimensional representations. Having obtained this matrix, the construction of the invariant follows from properties of ribbon Hopf (super)algebras and their representations. Here we consider the invariants of $(1,1)$ tangles for the following reason: for invariants derived from representations of quantum superalgebras with zero $q$-superdimension, the corresponding invariant is also zero. If the representation is irreducible, the quantum superalgebra symmetry of the procedure ensures that the invariant of $(1,1)$ tangles takes the form of some scalar multiple of the identity matrix. (See [35] for a discussion of this symmetry.) We take this scalar to be the invariant.

In this paper, we prove that the Links-Gould invariant is not able to distinguish between mutant links ( 4.8$)$, nor is it able to distinguish a knot from its inverse (i.e. from the knot obtained by reversing the orientation) (Proposition 3.2). However it is good at distinguishing some knots and links from their mirror images (see Propositions 22 and 4.1), and it is distinguished from the HOMFLY and Jones polynomials by this behaviour (see $\$ 4.5$ for specific examples). We provide many examples and a complete description of the state model for the invariant in abstract tensor form. This description of the invariant directly facilitates the construction of a computer program in MATHEMATICA for calculation of the invariant. 


\section{Construction of the $R$ Matrix}

We consider the family of four dimensional representations of the quantum superalgebra $U_{q}[g l(2 \mid 1)]$, which depend on a free complex parameter $\alpha$. This superalgebra has 7 simple generators $\left\{E^{1}{ }_{1}, E^{2}{ }_{2}, E^{3}{ }_{3}, E^{1}{ }_{2}, E^{2}{ }_{1}, E^{2}{ }_{3}, E^{3}{ }_{2}\right\}$ on which we define a $\mathbb{Z}_{2}$ grading in terms of the natural grading on the indices $[1]=[2]=0,[3]=1$ by:

$$
\left[E^{i}{ }_{j}\right]=[i]+[j] \quad(\bmod 2) .
$$

The $U_{q}[g l(2 \mid 1)]$ generators satisfy the commutation relations:

$$
\begin{aligned}
{\left[E^{1}{ }_{2}, E^{2}{ }_{1}\right] } & =\left[E^{1}{ }_{1}-E^{2}{ }_{2}\right]_{q} \\
\left\{E^{2}{ }_{3}, E^{3}{ }_{2}\right\} & =\left[E^{2}{ }_{2}+E^{3}{ }_{3}\right]_{q} \\
{\left[E^{i}{ }_{i}, E^{j}{ }_{k}\right] } & =\delta^{j}{ }_{i} E^{i}{ }_{k}-\delta^{i}{ }_{k} E^{j}{ }_{i}, \quad i, j, k=1,2,3,
\end{aligned}
$$

where $[$,$] and \{$,$\} denote the usual commutator and anticommutator, respectively and$ we have employed the $q$ bracket, defined for a wide class of objects $x$ by:

$$
[x]_{q} \triangleq \frac{q^{x}-q^{-x}}{q-q^{-1}} .
$$

Let $\{|i\rangle\}_{i=1}^{4}$ denote a basis for the four dimensional $U_{q}[g l(2 \mid 1)]$ module $V$. Consistent with the $\mathbb{Z}_{2}$ grading on $U_{q}[g l(2 \mid 1)]$, we grade the basis states by:

$$
[|1\rangle]=[|4\rangle]=0, \quad[|2\rangle]=[|3\rangle]=1 .
$$

We define a dual basis $\{\langle i|\}_{i=1}^{4}$; in component form, these are represented by the transpose complex conjugates of the original basis: $\left\langle i\left|=\overline{|i\rangle}^{t} \equiv\right| i\right\rangle^{\dagger}$. Then: $\langle i|| j\rangle \equiv\langle i \mid j\rangle=\delta_{i j}$. In terms of these dual bases, we define a representation $\pi$ of the $U_{q}[g l(2 \mid 1)]$ generators; their action on the basis vectors is given by:

$$
\begin{aligned}
& \pi\left(E^{1}{ }_{1}\right)=-|2\rangle\langle 2|-| 4\rangle\langle 4| \\
& \pi\left(E^{2}{ }_{2}\right)=-|3\rangle\langle 3|-| 4\rangle\langle 4| \\
& \pi\left(E^{3}{ }_{3}\right)=\alpha|1\rangle\langle 1|+(\alpha+1)(|2\rangle\langle 2|+| 3\rangle\langle 3|)+(\alpha+2)| 4\rangle\langle 4| \\
& \pi\left(E^{1}{ }_{2}\right)=-|3\rangle\langle 2| \\
& \pi\left(E^{2}{ }_{1}\right)=-|2\rangle\langle 3| \\
& \pi\left(E^{2}{ }_{3}\right)=[\alpha]_{q}^{1 / 2}|1\rangle\left\langle 3\left|-[\alpha+1]_{q}^{1 / 2}\right| 2\right\rangle\langle 4| \\
& \pi\left(E^{3}{ }_{2}\right)=[\alpha]_{q}^{1 / 2}|3\rangle\left\langle 1\left|-[\alpha+1]_{q}^{1 / 2}\right| 4\right\rangle\langle 2| .
\end{aligned}
$$


Associated with $U_{q}[g l(2 \mid 1)]$ there is a co-product structure $\left(\mathbb{Z}_{2}\right.$-graded algebra homomorphism) $\Delta: U_{q}[g l(2 \mid 1)] \rightarrow U_{q}[g l(2 \mid 1)] \otimes U_{q}[g l(2 \mid 1)]$ given by:

$$
\begin{aligned}
\Delta\left(E^{i}{ }_{i}\right) & =I \otimes E^{i}{ }_{i}+E^{i}{ }_{i} \otimes I, \quad i=1,2,3, \\
\Delta\left(E^{1}{ }_{2}\right) & =E^{1}{ }_{2} \otimes q^{-\frac{1}{2}\left(E^{1}{ }_{1}-E^{2}{ }_{2}\right)}+q^{\frac{1}{2}\left(E^{1}{ }_{1}-E^{2}{ }_{2}\right)} \otimes E^{1}{ }_{2} \\
\Delta\left(E^{2}{ }_{1}\right) & =E^{2}{ }_{1} \otimes q^{-\frac{1}{2}\left(E^{1}{ }_{1}-E^{2}{ }_{2}\right)}+q^{\frac{1}{2}\left(E^{1}{ }_{1}-E^{2}{ }_{2}\right)} \otimes E^{2}{ }_{1} \\
\Delta\left(E^{2}{ }_{3}\right) & =E^{2}{ }_{3} \otimes q^{-\frac{1}{2}\left(E^{2}{ }_{2}+E^{3}{ }_{3}\right)}+q^{\frac{1}{2}\left(E^{2}{ }_{2}+E^{3}{ }_{3}\right)} \otimes E^{2}{ }_{3} \\
\Delta\left(E^{3}{ }_{2}\right) & =E^{3}{ }_{2} \otimes q^{-\frac{1}{2}\left(E^{2}{ }_{2}+E^{3}{ }_{3}\right)}+q^{\frac{1}{2}\left(E^{2}{ }_{2}+E^{3}{ }_{3}\right)} \otimes E^{3}{ }_{2} .
\end{aligned}
$$

There exists another possible co-product structure: $\bar{\Delta}$, defined by $\bar{\Delta}=T \cdot \Delta$, where $T: U_{q}[g l(2 \mid 1)] \otimes U_{q}[g l(2 \mid 1)] \rightarrow U_{q}[g l(2 \mid 1)] \otimes U_{q}[g l(2 \mid 1)]$ is the twist map, defined for homogeneous elements $a, b \in U_{q}[g l(2 \mid 1)]$ :

$$
T(a \otimes b)=(-)^{[a][b]}(b \otimes a) .
$$

The tensor product module has the following decomposition with respect to the co-product for generic values of $\alpha$ :

$$
V \otimes V=V_{1} \oplus V_{2} \oplus V_{3}
$$

We construct symmetry adapted bases $\left\{\left|\Psi_{1}^{k}\right\rangle\right\}_{k=1}^{4}$ and $\left\{\left|\Psi_{3}^{k}\right\rangle\right\}_{k=1}^{4}$, for the spaces $V_{1}$ and $V_{3}$ respectively in terms of the basis elements of $V$ :

$$
\begin{aligned}
\left|\Psi_{1}^{1}\right\rangle & =|1\rangle \otimes|1\rangle \\
\left|\Psi_{2}^{1}\right\rangle & =\left(q^{\alpha}+q^{-\alpha}\right)^{-\frac{1}{2}}\left(q^{\alpha / 2}|1\rangle \otimes|2\rangle+q^{-\alpha / 2}|2\rangle \otimes|1\rangle\right) \\
\left|\Psi_{3}^{1}\right\rangle & =\left(q^{\alpha}+q^{-\alpha}\right)^{-\frac{1}{2}}\left(q^{\alpha / 2}|1\rangle \otimes|3\rangle+q^{-\alpha / 2}|3\rangle \otimes|1\rangle\right) \\
\left|\Psi_{4}^{1}\right\rangle & =\left(q^{\alpha}+q^{-\alpha}\right)^{-\frac{1}{2}}[2 \alpha+1]_{q}^{-\frac{1}{2}} \times \\
& \left.\quad[\alpha+1]_{q}^{\frac{1}{2}}\left(q^{\alpha}|1\rangle \otimes|4\rangle+q^{-\alpha}|4\rangle \otimes|1\rangle\right)+[\alpha]_{q}^{\frac{1}{2}}\left(q^{\frac{1}{2}}|2\rangle \otimes|3\rangle-q^{-\frac{1}{2}}|3\rangle \otimes|2\rangle\right)\right] \\
\left|\Psi_{1}^{3}\right\rangle & =\left(q^{\alpha+1}+q^{-\alpha-1}\right)^{-\frac{1}{2}}[2 \alpha+1]_{q}^{-\frac{1}{2}} \times \\
{\left[[\alpha]_{q}^{\frac{1}{2}}\right.} & \left.\left(q^{\alpha+1}|4\rangle \otimes|1\rangle+q^{-\alpha-1}|1\rangle \otimes|4\rangle\right)+[\alpha+1]_{q}^{\frac{1}{2}}\left(q^{-\frac{1}{2}}|3\rangle \otimes|2\rangle-q^{\frac{1}{2}}|2\rangle \otimes|3\rangle\right)\right] \\
\left|\Psi_{2}^{3}\right\rangle & =\left(q^{\alpha+1}+q^{-\alpha-1}\right)^{-\frac{1}{2}}\left(q^{(\alpha+1) / 2}|4\rangle \otimes|2\rangle+q^{-(\alpha+1) / 2}|2\rangle \otimes|4\rangle\right) \\
\left|\Psi_{3}^{3}\right\rangle & =\left(q^{\alpha+1}+q^{-\alpha-1}\right)^{-\frac{1}{2}}\left(q^{(\alpha+1) / 2}|4\rangle \otimes|3\rangle+q^{-(\alpha+1) / 2}|3\rangle \otimes|4\rangle\right) \\
\left|\Psi_{4}^{3}\right\rangle & =|4\rangle \otimes|4\rangle .
\end{aligned}
$$

Dual bases $\left\{\left\langle\Psi_{1}^{k}\right|\right\}_{k=1}^{4}$ and $\left\{\left\langle\Psi_{3}^{k}\right|\right\}_{k=1}^{4}$, are found from the definitions:

$$
\begin{aligned}
\left\langle\Psi_{j}^{k}\right| & =\left|\Psi_{j}^{k}\right\rangle^{\dagger}, \quad k=1,3, \quad j=1, \ldots, 4, \\
(|i\rangle \otimes|j\rangle)^{\dagger} & =(-)^{[|i\rangle][j\rangle]}(\langle i| \otimes\langle j|), \quad i, j=1, \ldots, 4 .
\end{aligned}
$$


Now, the general form of the basis vectors $\left|\Psi_{j}^{k}\right\rangle$ is:

$$
\left|\Psi_{j}^{k}\right\rangle=\sum_{m} \theta_{m}^{k j}\left(\left|x_{m}^{k j}\right\rangle \otimes\left|y_{m}^{k j}\right\rangle\right)
$$

where the $\theta_{m}^{k j}$ are in general complex scalar functions of $q$ and $\alpha$. From (2) and (3), and choosing the parameters $q$ and $\alpha$ to be real and positive, the duals of these vectors are given by:

$$
\left\langle\Psi_{j}^{k}\right|=\sum_{m}(-)^{\left[\left|x_{m}^{k j}\right\rangle\right]\left[\left|y_{m}^{k j}\right\rangle\right]} \theta_{m}^{k j}\left(\left\langle x_{m}^{k j}\right| \otimes\left\langle y_{m}^{k j}\right|\right) .
$$

As the $R$ matrix is unique, analytic continuation makes our final results valid for any complex $q$ and $\alpha$. For the duals, we obtain:

$$
\begin{aligned}
\left\langle\Psi_{1}^{1}\right| & =\langle 1| \otimes\langle 1| \\
\left\langle\Psi_{2}^{1}\right| & =\left(q^{\alpha}+q^{-\alpha}\right)^{-\frac{1}{2}}\left(q^{\frac{1}{2} \alpha}\langle 1| \otimes\langle 2|+q^{-\frac{1}{2} \alpha}\langle 2| \otimes\langle 1|\right) \\
\left\langle\Psi_{3}^{1}\right| & =\left(q^{\alpha}+q^{-\alpha}\right)^{-\frac{1}{2}}\left(q^{\frac{1}{2} \alpha}\langle 1| \otimes\langle 3|+q^{-\frac{1}{2} \alpha}\langle 3| \otimes\langle 1|\right) \\
\left\langle\Psi_{4}^{1}\right| & =\left(q^{\alpha}+q^{-\alpha}\right)^{-\frac{1}{2}}[2 \alpha+1]_{q}^{-\frac{1}{2}} \times \\
& {\left[[\alpha+1]_{q}^{\frac{1}{2}}\left(q^{\alpha}\langle 1| \otimes\langle 4|+q^{-\alpha}\langle 4| \otimes\langle 1|\right)-[\alpha]_{q}^{\frac{1}{2}}\left(q^{\frac{1}{2}}\langle 2| \otimes\langle 3|-q^{-\frac{1}{2}}\langle 3| \otimes\langle 2|\right)\right] } \\
\left\langle\Psi_{1}^{3}\right| & =\left(q^{\alpha+1}+q^{-\alpha-1}\right)^{-\frac{1}{2}}[2 \alpha+1]_{q}^{-\frac{1}{2}} \times \\
{[} & {\left.[\alpha]_{q}^{\frac{1}{2}}\left(q^{\alpha+1}\langle 4| \otimes\langle 1|+q^{-\alpha-1}\langle 1| \otimes\langle 4|\right)-[\alpha+1]_{q}^{\frac{1}{2}}\left(q^{-\frac{1}{2}}\langle 3| \otimes\langle 2|-q^{\frac{1}{2}}\langle 2| \otimes\langle 3|\right)\right] } \\
\left\langle\Psi_{2}^{3}\right| & =\left(q^{\alpha+1}+q^{-\alpha-1}\right)^{-\frac{1}{2}}\left(q^{\frac{1}{2}(\alpha+1)}\langle 4| \otimes\langle 2|+q^{-\frac{1}{2}(\alpha+1)}\langle 2| \otimes\langle 4|\right) \\
\left\langle\Psi_{3}^{3}\right| & =\left(q^{\alpha+1}+q^{-\alpha-1}\right)^{-\frac{1}{2}}\left(q^{\frac{1}{2}(\alpha+1)}\langle 4| \otimes\langle 3|+q^{-\frac{1}{2}(\alpha+1)}\langle 3| \otimes\langle 4|\right) \\
\left\langle\Psi_{4}^{3}\right| & =\langle 4| \otimes\langle 4| .
\end{aligned}
$$

From the basis vectors $\left|\Psi_{j}^{k}\right\rangle$ and their duals $\left\langle\Psi_{j}^{k}\right|$ for $V_{1}$ and $V_{3}$, we construct projectors $P_{1}$ and $P_{3}$, defined by:

$$
P_{1}=\sum_{k=1}^{4}\left|\Psi_{1}^{k}\right\rangle\left\langle\Psi_{1}^{k}\left|, \quad P_{3}=\sum_{k=1}^{4}\right| \Psi_{3}^{k}\right\rangle\left\langle\Psi_{3}^{k}\right| .
$$

Note that the multiplication operation on the graded space $V \otimes V$ is given by:

$$
(|i\rangle \otimes|j\rangle)(\langle k| \otimes\langle l|)=(-)^{[|j\rangle][\langle k|]}(|i\rangle\langle k|\otimes| j\rangle\langle l|), \quad i, j, k, l=1,2,3,4 .
$$

Now let $I$ be the identity operator on $V \otimes V$, viz: $I=\sum_{i j=1}^{4} e^{i}{ }_{i} \otimes e^{j}{ }_{j}$, where $e^{k}{ }_{l}=|k\rangle\langle l|$ is an elementary rank 2 tensor. As we have $P_{1}+P_{2}+P_{3}=I$, we thus do not need to explicitly construct $P_{2}$ (or even a basis for $V_{2}$ ); we simply set:

$$
P_{2}=I-P_{1}-P_{3}
$$


Where $g$ is a classical Lie superalgebra, the corresponding quantum superalgebra $U_{q}[g]$ admits a universal $R$ matrix $R \in U_{q}[g] \otimes U_{q}[g]$ satisfying (among other relations):

$$
\begin{aligned}
R \Delta(a) & =\bar{\Delta}(a) R, & & \forall a \in U_{q}[g], \\
R_{12} R_{13} R_{23} & =R_{23} R_{13} R_{12}, & & \text { in } U_{q}[g] \otimes U_{q}[g] \otimes U_{q}[g],
\end{aligned}
$$

where the subscripts refer to the embedding of $R$ acting on the triple tensor product space. From any representation of $U_{q}[g]$, one may obtain a tensor solution of (6) by replacing the superalgebra elements with their matrix representatives. Similarly to (4), multiplication of tensor products of matrices $a, b, c, d$ is governed by:

$$
(a \otimes b)(c \otimes d)=(-)^{[b][c]}(a c \otimes b d), \quad \text { homogeneous } b, c .
$$

We introduce the graded permutation operator $P$ on the tensor product space $V \otimes V$, defined for graded basis vectors $v^{k}, v^{l} \in V$ by:

$$
P\left(v^{k} \otimes v^{l}\right)=(-)^{[k][l]}\left(v^{l} \otimes v^{k}\right)
$$

and extended by linearity. (We use the shorthand $\left[v^{k}\right] \equiv[k]$.) With this, we define:

$$
\sigma=P R
$$

which can be shown to satisfy the equation:

$$
(\sigma \otimes I)(I \otimes \sigma)(\sigma \otimes I)=(I \otimes \sigma)(\sigma \otimes I)(I \otimes \sigma)
$$

From [26], we have (with a slight change of notation and a convenient choice of normalisation):

$$
\sigma=q^{-2 \alpha} P_{1}-P_{2}+q^{2 \alpha+2} P_{3}
$$

Using (5), this simplifies to:

$$
\sigma=\left(1+q^{-2 \alpha}\right) P_{1}+\left(1+q^{2 \alpha+2}\right) P_{3}-I .
$$

From the above form of $\sigma$, it is straightforward to deduce that $\sigma$ satisfies the polynomial identity:

$$
q^{-1} \sigma^{3}+\left(q^{-1}-q^{-2 \alpha-1}-q^{2 \alpha+1}\right) \sigma^{2}+\left(q-q^{-2 \alpha-1}-q^{2 \alpha+1}\right) \sigma+q I=0 .
$$

The above skein relation may be used to evaluate the invariant in some cases, but not all since it is of third order. The invariant may also be directly evaluated for a class of links using quantum superalgebra theoretic results [12]. 
We will represent rank 2 tensors as matrices, that is, the elementary rank 2 tensor $e_{k}^{i}$ is represented by the elementary $(4 \times 4)$ matrix $e_{i, k}$. We adopt the (standard) convention that the elementary rank 4 tensor $e^{i j}{ }_{k l}=e^{i}{ }_{k} \otimes e^{j}{ }_{l}$ is constructed by insertion of a copy of the elementary rank 2 tensor $e^{j}{ }_{l}$ at each location of $e^{i}{ }_{k}$ (i.e. each element of $e^{i}{ }_{k}$ is multiplied by the whole of $e^{j}$ ). This means that $e^{i j} k$ is represented by the elementary $(16 \times 16)$ matrix $e_{4(i-1)+j, 4(k-1)+l}$.

Let $A$ be an arbitrary graded rank 4 tensor acting on $V \otimes V$, then for scalar coefficients $A^{i j}{ }_{k l}$ :

$$
A=\sum_{i j k l} A_{k l}^{i j}\left(e_{k}^{i} \otimes e^{j}\right)
$$

Our convention then tells us that the coefficient $A^{i j}{ }_{k l}$ is the $(4(i-1)+j, 4(k-1)+l)$ entry of $A$, written explicitly:

$$
A^{i j}{ }_{k l} \mapsto A_{4(i-1)+j, 4(k-1)+l}
$$

We wish to remove the grading on $V$, and convert the matrix representing $\sigma$ to its ungraded counterpart. Recall that basis vectors $v^{k}$ satisfy $e^{i}{ }_{j} v^{k}=\delta^{k}{ }_{j} v^{i}$, hence the action of $A$ on $V \otimes V$ is:

$$
\begin{aligned}
A\left(v^{k} \otimes v^{l}\right) & =\sum_{i j m n} A^{i j}{ }_{m n}\left(e^{i}{ }_{m} \otimes e^{j}{ }_{n}\right)\left(v^{k} \otimes v^{l}\right) \\
& =\sum_{i j m n} A^{i j}{ }_{m n}(-)^{[k]([j]+[n])}\left(e^{i}{ }_{m} v^{k} \otimes e^{j}{ }_{n} v^{l}\right) \\
& =\sum_{i j m n} A^{i j}{ }_{m n}(-)^{[k]([j]+[n])}\left(\delta^{k}{ }_{m} v^{i} \otimes \delta^{l}{ }_{n} v^{j}\right) \\
& =\sum_{i j m n} A^{i j}{ }_{m n}(-)^{[k]([j]+[n])} \delta^{k}{ }_{m} \delta_{n}^{l}\left(v^{i} \otimes v^{j}\right) \\
& =\sum_{i j} A^{i j}{ }_{k l}(-)^{[k]([j]+[l])}\left(v^{i} \otimes v^{j}\right) .
\end{aligned}
$$

Now, in this sum, the parity factor is constructed from the degrees of vectors; in the ungraded case, there would be no such factor, indeed we would have:

$$
\bar{A}\left(v^{k} \otimes v^{l}\right)=\sum_{i j} \bar{A}_{k l}^{i j}\left(v^{i} \otimes v^{j}\right) .
$$

This motivates us to set:

$$
\bar{A}_{k l}^{i j}=(-)^{[k]([j]+[l])} A^{i j}{ }_{k l} .
$$

Under these conventions, the explicit form of $\sigma$ is presented (as a matrix!) in $\S$. 


\section{Knot Theory}

\subsection{Link Examples}

In Table 1, we list the links to be studied. (By the term 'knot', we intend a link of one component.) We use the well-known notation of Alexander and Briggs (1926) [3], the data being abstracted from [1], itself citing [36] and [8] (beware that the tables in this latter article are presented in microfiche form only).

\begin{tabular}{||l|c|l|l||}
\hline \hline \multicolumn{1}{||c|}{$K$} & $w(K)$ & \multicolumn{1}{|c|}{ Chiral? } & \multicolumn{1}{|c||}{ Invertible? } \\
\hline \hline $0_{1}$ (Unknot) & 0 & No (trivial) & Yes (trivial) \\
\hline $2_{1}^{2}$ (Hopf Link) & 2 & No (trivial) & Yes (trivial) \\
\hline $3_{1}$ (Trefoil) & 3 & $Y e s$ [1, p 176] & Yes (trivial) \\
\hline $4_{1}$ (Figure Eight) & 0 & $\begin{array}{l}\text { No (11, p 14]; see } \\
\text { [17, p 198] for an } \\
\text { elegant graphical } \\
\text { proof) }\end{array}$ & $\begin{array}{l}\text { Yes (as } 8_{17} \text { is } \\
\text { thertible knot) }\end{array}$ \\
\hline $5_{1}^{2}$ (Whitehead Link) & 1 & $Y e s$ [16, pp 49-50] & Yes \\
\hline $8_{17}$ & 0 & No [16, p 455] & No [19, p 162] \\
\hline $9_{42}$ & 1 & $Y e s$ [18, p 218] & Yes \\
\hline $10_{48}$ & 0 & $Y e s$ [18, p 218] & Yes \\
\hline \hline
\end{tabular}

Table 1: Data for the links to be investigated, including their Alexander-Briggs (and common) names, their writhes $w(K)$, and whether they are chiral and invertible. Diagrams of the links are presented in Figures 9 to 13 .

\subsection{Reflection and Inversion - Chirality and Invertibility}

Throughout, we shall write "=" to denote ambient isotopy of link diagrams, meaning that they are equivalent under the Reidemeister moves (original: [33], but see, e.g. [18]). We shall use the following definitions, but the reader must be aware that conflicting terminology appears in the literature. 
Reflection: We shall denote by $K^{*}$ the mirror image (or reflection) of a knot $K$. A knot is chiral if it is distinct from its mirror image; i.e. there are actually two distinct knots with the same name, $K^{*} \neq K$, e.g. the trefoil knot is chiral: $\left(3_{1}\right)^{*} \neq 3_{1}$. Note that this definition doesn't require an orientation. A knot is amphichiral if it is ambient isotopic to its mirror image, i.e. $K^{*}=K$.

The HOMFLY] (and hence the Jones) polynomial and the Kauffman polynomial can distinguish many (but not all) knots from their reflections. The first chiral knot that neither the HOMFLY nor the Kauffman polynomial can distinguish is $9_{42}$, i.e. $9_{42}^{*} \neq 9_{42}$, but the polynomials are equal. Similarly, the knot $10_{48}$ is chiral, but the HOMFLY polynomial fails to detect this, although the Kauffman does detect it 18, p 218] (wrongly labeled $10_{79}$ ).

Inversion: Assign an orientation to a knot. Denote the inverse of a knot $K$ by $K^{-1}$, obtained by reversing the orientation. Whilst this is a simple concept for a knot, there are of course many possibilities for the reversal of only some components of oriented, multi-component links; we shall not go into these here.

Commonly, $K=K^{-1}$, and we say that $K$ is invertible. For example, the trefoil knot is invertible $\left(3_{1}\right)^{-1}=3_{1}$. Less commonly, $K \neq K^{-1}$, and we say that $K$ is noninvertible. The first example of a noninvertible (prime) knot is $8_{17}$.

Both the reflection and the inverse are automorphisms of order two, i.e. $\left(K^{*}\right)^{*}=K$ and $\left(K^{-1}\right)^{-1}=K$. The notions may of course be combined, we obtain: $\left(K^{*}\right)^{-1}=\left(K^{-1}\right)^{*}$.

To illustrate, using the Trefoil Knot $3_{1}$ (see Figure 9). We have two equivalence classes: $3_{1}=\left(3_{1}\right)^{-1}$ and $\left(3_{1}\right)^{*}=\left(\left(3_{1}\right)^{-1}\right)^{*}=\left(\left(3_{1}\right)^{*}\right)^{-1}$.

\subsection{Abstract Tensor Conventions}

By a 'positive oriented' or 'right-handed' crossing, we shall intend a crossing such that if the thumb of the right hand points in the direction of one of the arrows, the fingers of the right hand will point in the direction of the other arrow. The opposite situation is naturally called a 'negative oriented' or 'left-handed' crossing.

If the two outward-pointing arrows of a positive oriented crossing are pointed upwards, then we shall label the components of the crossing with indices $a$ in top left, $b$ in bottom left, $c$ in top right, and $d$ in bottom right, and associate with the crossing the (rank 4) tensor $\sigma_{b d}^{a c}$, where the position of the indices in the tensor corresponds with the positioning of the labels in the crossing. The inverse of $\sigma$ will represent a negative oriented crossing, with the convention on the indices being the same as that of $\sigma$. A diagram of $\sigma$ and $\sigma^{-1}$ is provided in Figure 1 .

${ }^{1}$ The HOMFLY polynomial is named by the conjunction of the initials of six of its discoverers $[10$, omitting those ("P" and "T") of two independent discoverers [32]. Przytycki, the omitted "P", has furthered the entymological spirit with the suggestion "FLYPMOTH" [31, p 256], which includes all the discoverers and has a muted reference to the "flyping" operation of the Tait, Kirkwood and Little - the original compilers of knot tables. (Another possibility is the letter sequence "HOMFLYPT".) Bar-Natan (Prasolov and Sossinsky [30, p 36] cite Bar-Natan [4], who cites "L Rudulph") goes further, adding a "U" for good measure, to account for any unknown discoverers, yielding the unpalatable "LYMPHTOFU"! 

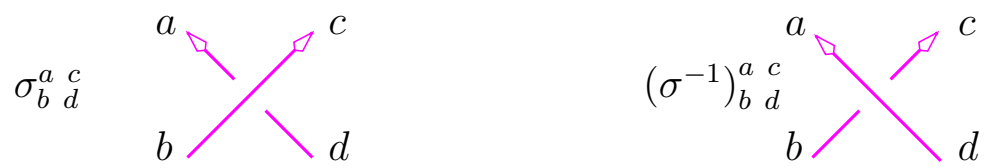

Figure 1: Definition of the tensors $\sigma$ and $\sigma^{-1}$ representing positive oriented and negative oriented crossings with upward pointing arrows, respectively.

We shall also require four (rank 2) tensors (i.e. genuine matrices) to represent all possible horizontally-oriented half-loops. We shall call these 'cap' and 'cup' matrices, and label them with the suggestive $\Omega^{ \pm}$and $\mho^{ \pm}$, e.g. $\Omega^{+}$is the upper loop with arrow pointing right. A diagram is provided in Figure 2 .
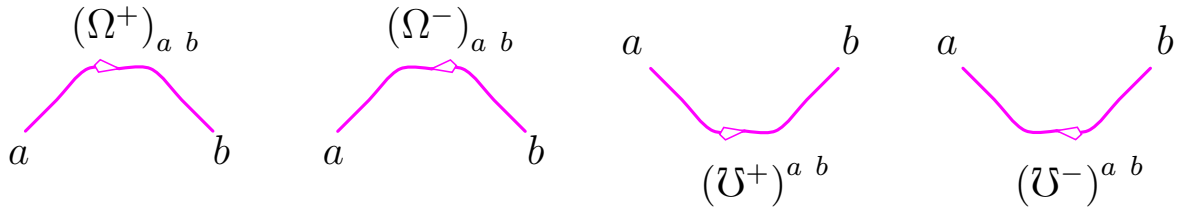

Figure 2: Definition of the tensors (matrices) $\Omega^{ \pm}$and $\mho^{ \pm}$, representing all possibilities of horizontally-aligned half-loops.

With these basic tensors $\sigma, \sigma^{-1}, \Omega^{ \pm}$and $\mho^{ \pm}$, we may evaluate an invariant for any particular link. However, this direct procedure tends to be computationally expensive, and parts of the computation are often repeated, so in practice, we define auxiliary symbols. We shall use the notation $X$ to represent a rank 4 tensor such as $\sigma$ or $\sigma^{-1}$ with parallel pointing arrows (i.e. a 'channel' crossing in the terminology of Kauffman [18, p 76].)

The primary auxiliary tensors used are listed below; secondary ones will be mentioned where necessary. The Einstein summation convention is used throughout.

- The first auxiliary symbols are those of crossings that have been 'twisted' relative to $\sigma$ and $\sigma^{-1}$. The left, right, and upside-down-twisted versions of $X$ will be called $X_{l}, X_{r}$ and $X_{d}$ respectively. They are defined in the following manner:

$$
\begin{aligned}
& \left(X_{l}\right)_{b d}^{a c} \triangleq X_{d h}^{e a} \cdot\left(\Omega^{-}\right)_{b e} \cdot\left(\mho^{-}\right)^{h c} \\
& \left(X_{r}\right)_{b d}^{a c} \triangleq X_{f b}^{c g} \cdot\left(\mho^{+}\right)^{a f} \cdot\left(\Omega^{+}\right)_{g d} \\
& \left(X_{d}\right)_{b d}^{a c} \triangleq X_{f h}^{e g} \cdot\left(\mho^{+}\right)^{a h} \cdot\left(\Omega^{+}\right)_{g b} \cdot\left(\mho^{+}\right)^{c f} \cdot\left(\Omega^{+}\right)_{e d} .
\end{aligned}
$$

Observe that $X_{d}$ is a 'channel' crossing, whilst $X_{l}$ and $X_{r}$ are 'cross-channel' crossings. Diagrams are found in Figures 3 and 4 .

- The next set of auxiliary symbols represent $p$ copies of the same crossing $X$ (for any channel crossing $X$ ) atop one another (see Figure 5). They are defined recursively in the following manner:

$$
\left(X^{p+1}\right)_{b d}^{a c} \triangleq X_{e f}^{a c} \cdot\left(X^{p}\right)_{b d}^{e f}, \quad p=1,2, \ldots
$$



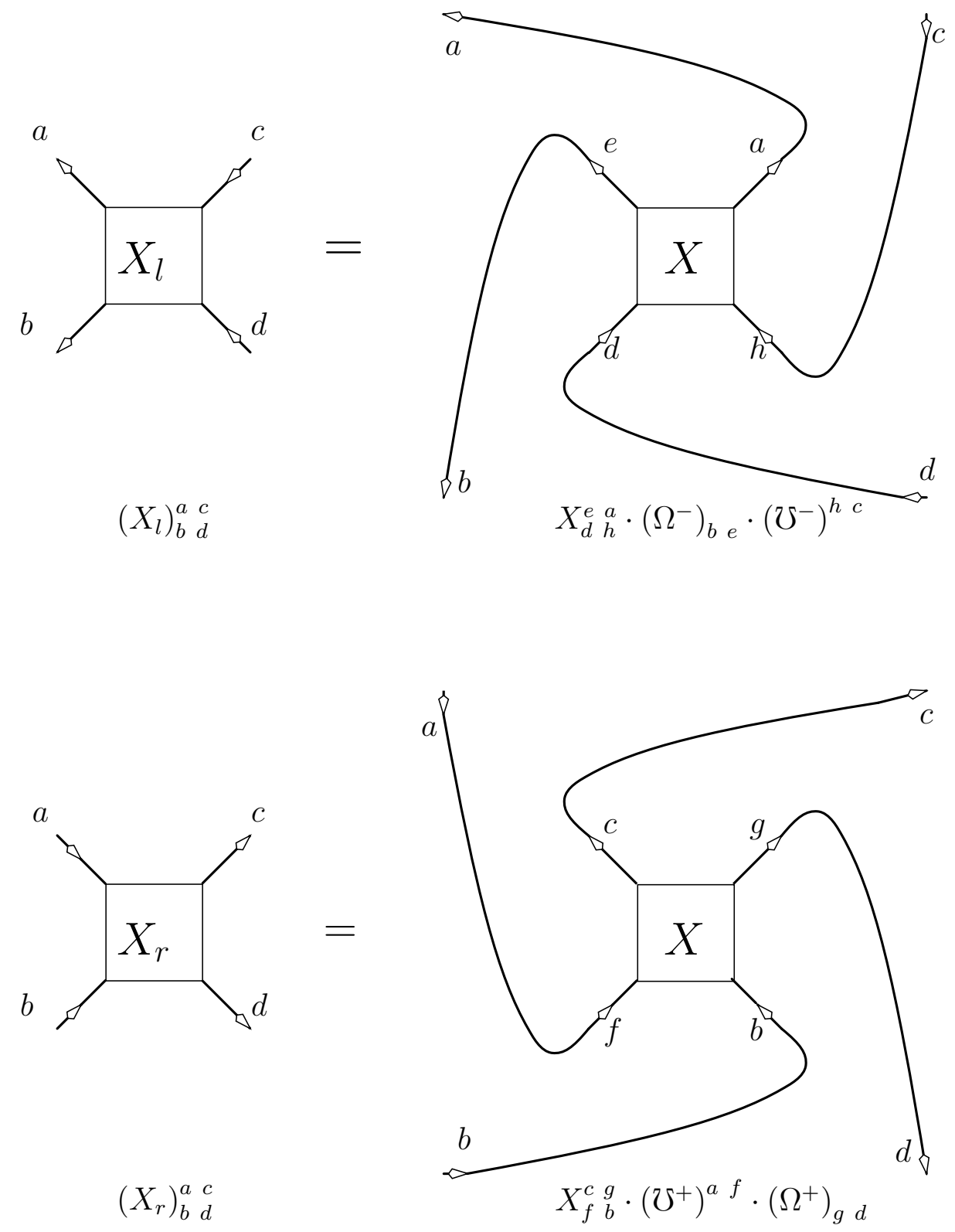

Figure 3: The primary auxiliary tensors $X_{l}$ and $X_{r}$, where $X$ is one of $\sigma$ or $\sigma^{-1}$. 

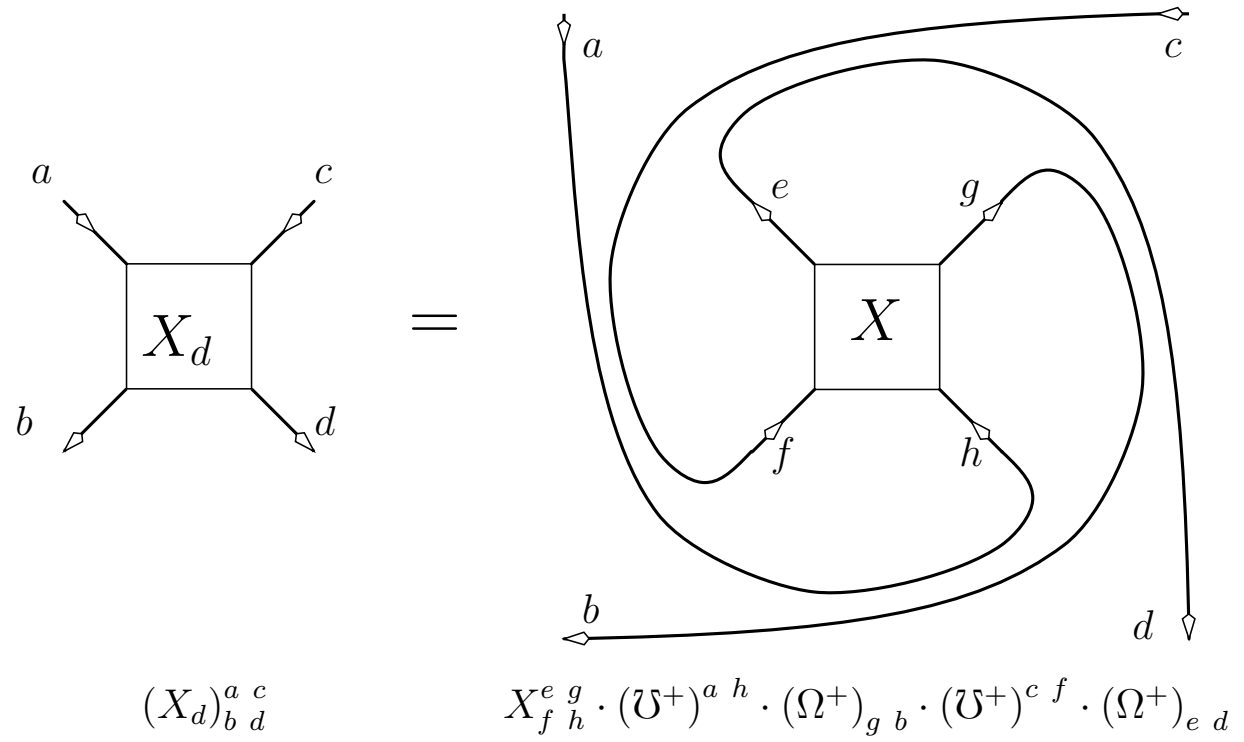

Figure 4: The primary auxiliary tensor $X_{d}$, where $X$ is one of $\sigma$ or $\sigma^{-1}$.

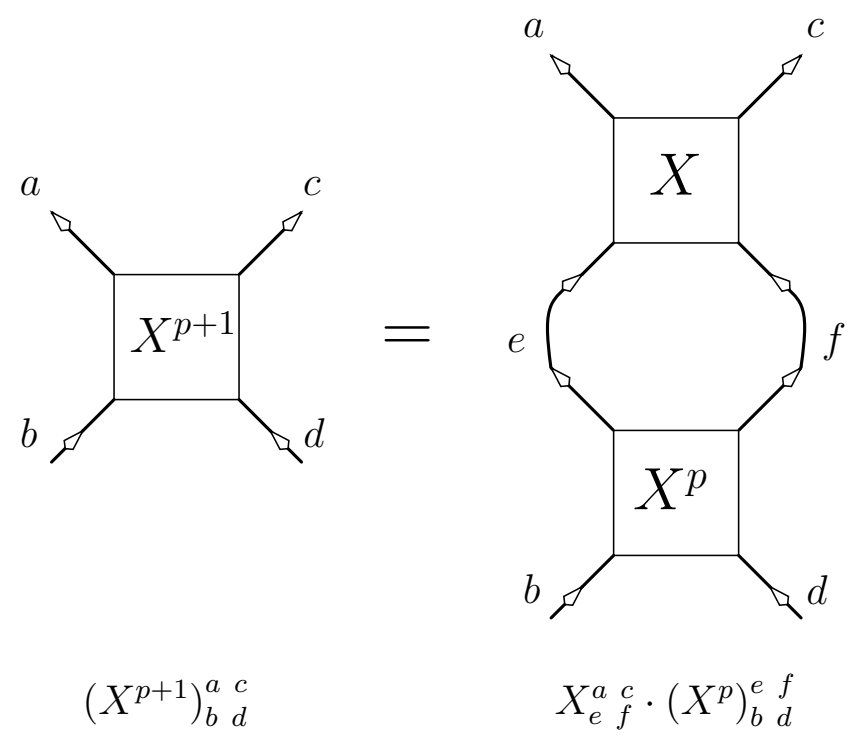

Figure 5: The primary auxiliary tensors $X^{p+1}$ in terms of $X$ and $X^{p} ; X$ is one of $\sigma$ or $\sigma^{-1}$. If all arrows are reversed, then the definition also holds for $X$ being $\sigma_{d}$ or $\sigma_{d}^{-1}$; that is, any channel crossing. 
- The third set of frequently-encountered patterns are where a crossing $X$ is to the left or right of its own 'upside-downness' $X_{d}$. That is, fix an $X$ as either $\sigma$ or $\sigma^{-1}$, and examine the patterns formed from juxtaposing $X$ and $X_{d}$. They are defined in the following manner:

$$
\begin{aligned}
& \left(X_{d} X\right)_{b d}^{a c} \triangleq\left(X_{d}\right)_{b f}^{a e} \cdot X_{h d}^{g c} \cdot\left(\Omega^{-}\right)_{e g} \cdot\left(\mho^{+}\right)^{f h} \\
& \left(X X_{d}\right)_{b d}^{a c} \triangleq X_{b f}^{a e} \cdot\left(X_{d}\right)_{h d}^{g c} \cdot\left(\Omega^{+}\right)_{e g} \cdot\left(\mho^{-}\right)^{f h} .
\end{aligned}
$$

A diagram is found in Figure 6 .
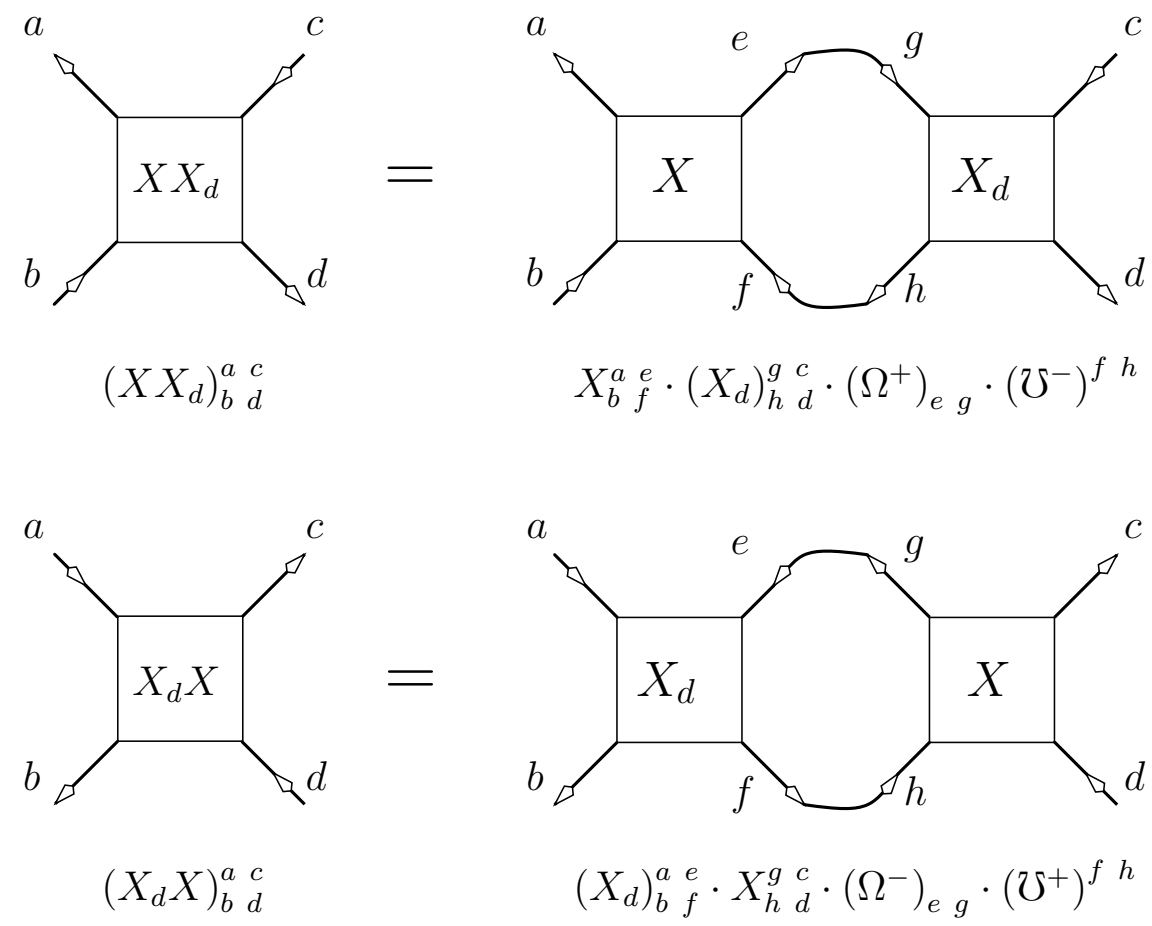

Figure 6: The primary auxiliary tensors $X_{d} X$ and $X X_{d} ; X$ is one of $\sigma$ or $\sigma^{-1}$.

- The final set of frequently-encountered patterns are where a crossing $X_{l}$ is placed atop above a crossing $X_{r}$ (or vice-versa). We obtain:

$$
\begin{aligned}
& \left(X_{l} X_{r}\right)_{b d}^{a c} \triangleq\left(X_{l}\right)_{e f}^{a c} \cdot\left(X_{r}\right)_{b d}^{e f}
\end{aligned}
$$

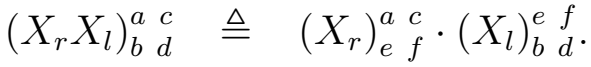

A diagram is found in Figure 7. A moment's thought demonstrates that the diagram for $X_{l} X_{r}$ is a right rotation of the diagram for $X_{d} X$. In fact, we have the identity:

$$
\left(X_{l} X_{r}\right)_{b d}^{a c}=\left(X_{d} X\right)_{d h}^{e a} \cdot\left(\mho^{+}\right)^{h c} \cdot\left(\Omega^{+}\right)_{b e},
$$

although in practice we shall not use it. (A diagram parallel to Figure 3 would demonstrate this.) 


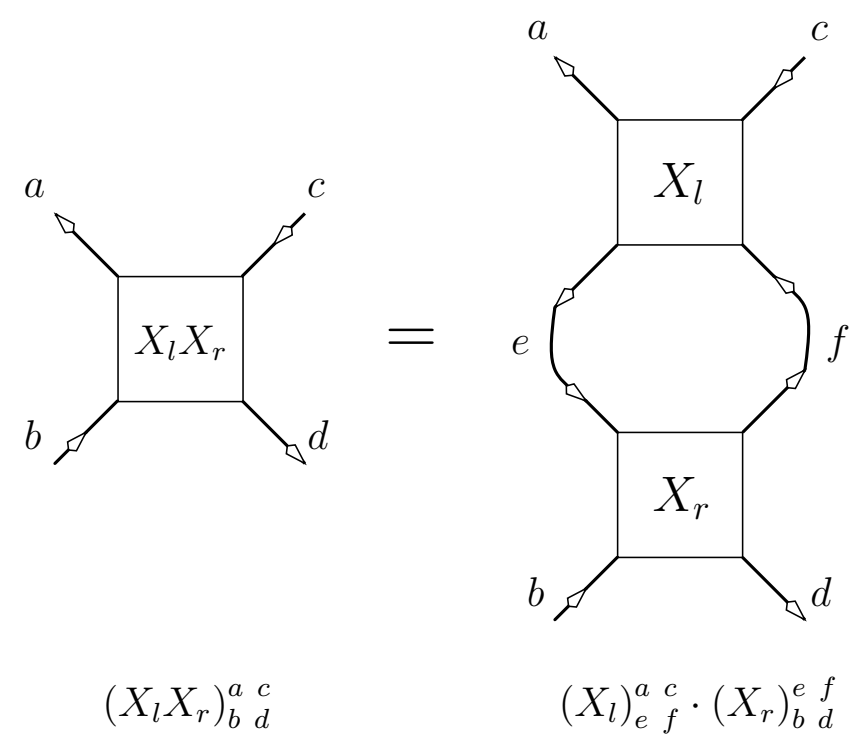

Figure 7: The primary auxiliary tensor $X_{l} X_{r} ; X$ is one of $\sigma$ or $\sigma^{-1} . X_{r} X_{l}$ is obtained by swapping every $r$ and $l$ in this diagram.

\subsection{The Effects of Reflection and Inversion on the Tensors}

Reflection: Let $K^{*}$ be the reflection of a tangle $K$; and say that we have constructed a tensor representing $K$. Every positive (respectively negative) crossing in $K$ will have been replaced by the equivalent negative (respectively positive) crossing in $K^{*}$. Thus, the tensor corresponding to $K^{*}$ will be that of $K$ with every $\sigma$ replaced by $\sigma^{-1}$, and every $\sigma^{-1}$ replaced by $\sigma$. This carries through to the auxiliary tensors; i.e. $\sigma_{d} \sigma$ will be replaced with $\sigma_{d}^{-1} \sigma^{-1}$, etc. The caps $\Omega^{ \pm}$and cups $\mho^{ \pm}$will remain unchanged.

From the uniqueness [20] of the universal $R$ matrix for any quantum superalgebra the following relation holds (for appropriate normalisation):

$$
R^{-1}(q)=R\left(q^{-1}\right)
$$

which in turn leads to the relation

$$
\sigma^{-1}(q)=P \sigma\left(q^{-1}\right) P
$$

Thus, up to a basis transformation, $\sigma$ and $\sigma^{-1}$ are interchangeable by the change of variable $q \mapsto q^{-1}$. It then follows that the invariant for $K^{*}$ is obtainable from that of $K$ by the same change of variable, which leads to the following:

\section{Proposition 3.1}

If $K$ is amphichiral then the invariant $L G_{K}$ is palindromic.

\footnotetext{
${ }^{2}$ We intend "palindromic" to mean that the polynomial is invariant under the mapping $q \mapsto q^{-1}$.
} 
Inversion: Again, if $K^{-1}$ is the inverse of $K$, then every arrow in $K$ will have been replaced with an arrow in the opposite direction. The tensor corresponding to $K^{-1}$ will thus have the following changes: For the crossings, where $X$ is either $\sigma$ or $\sigma^{-1}$, interchange $X \Longleftrightarrow X_{d}$ and $X_{l} \Longleftrightarrow X_{r}$; and for the caps and cups, interchange only the signs, i.e. $\Omega^{ \pm} \Longleftrightarrow \Omega^{\mp}$ and $\mho^{ \pm} \Longleftrightarrow \mho^{\mp}$.

This has the effect that the tensor representing $K$ is replaced by the dual tensor acting on the dual space [35]. Recalling that the tensors representing $(1,1)$ tangles act as scalar multiples of the identity on $V$, then the dual tensor has exactly the same form, from which we conclude:

\section{Proposition 3.2}

A knot invariant derived from an irreducible representation of a quantum (super)algebra is unable to detect inversion.

\subsection{Abstract Tensor Expressions for the Example Links}

We list the abstract tensors $\left(T_{K}\right)_{x}^{y}$ that represent the $(1,1)$-tangle (open diagram) forms of the example links. In each case, the indices $x$ and $y$ are the lower and upper loose ends of the tangle in question. The Links-Gould invariant is then formed by setting $x$ and $y$ to be the same, i.e.

$$
L G_{K}\left(q, p=q^{\alpha}\right) \triangleq\left(T_{K}\right)_{i}^{i}
$$

(no sum on $i$ ), for any allowable index $i$. We typically choose $i=1$. Our invariant does not need to be writhe-normalised, due to the choice of normalisation of $\sigma$ and the cap and cup matrices $\Omega^{ \pm}$and $\mho^{ \pm}$. Figure 8 depicts removal of a loop from a diagram.
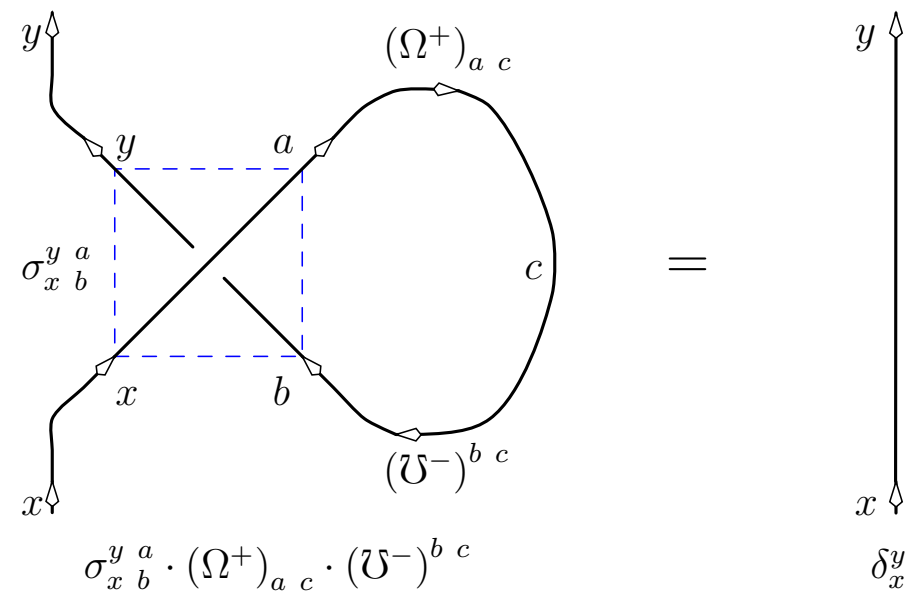

Figure 8: Removal of a Single, Positive Loop.

Braid presentations for the example knots are taken from [14, pp 109-110] and [15, pp 381386]. 
$\mathbf{0}_{\mathbf{1}}$ (Unknot): A braid presentation is the trivial $e \in B_{1}$. As the unclosed tangle representing the Unknot is rather meaningless, we use simply $\left(T_{0_{1}}\right)_{x}^{y} \triangleq \delta_{x}^{y}$.

$\mathbf{2}_{1}^{2}$ (Hopf Link): A braid presentation is $\sigma_{1}^{2} \in B_{2}$. Diagrams pertaining to the Hopf Link and Trefoil are found in Figure 9.

$$
\left(T_{2_{1}^{2}}\right)_{x}^{y} \triangleq\left(\sigma^{2}\right)_{x b}^{y a} \cdot\left(\Omega^{+}\right)_{a c} \cdot\left(\mho^{-}\right)^{b c}
$$

$\mathbf{3}_{\mathbf{1}}$ (Trefoil): A braid presentation is $\sigma_{1}{ }^{3} \in B_{2}$. This knot has also been called the overhand knot (as that is how it is tied) and the cloverleaf knot [7, pp 3-4].

$$
\left(T_{3_{1}}\right)_{x}^{y} \triangleq\left(\sigma^{3}\right)_{x b}^{y a} \cdot\left(\Omega^{+}\right)_{a c} \cdot\left(\mho^{-}\right)^{b c}
$$
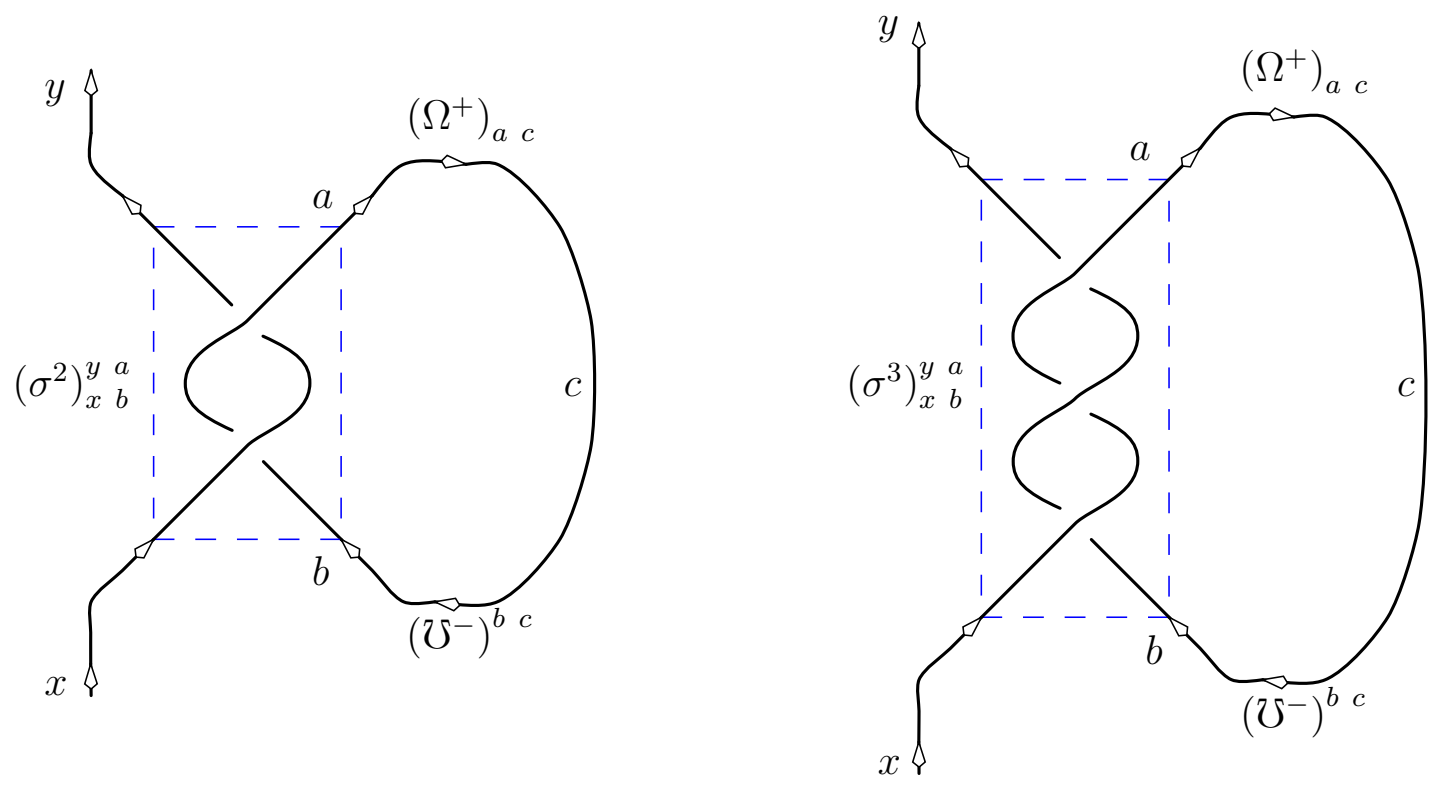

Figure 9: Tangle form of $2_{1}^{2}$ (the Hopf Link) and $3_{1}$ (the positive Trefoil). 
$4_{1}$ (Figure Eight): A braid presentation is $\left(\sigma_{1} \sigma_{2}^{-1}\right)^{2} \in B_{3}$, and a diagram is found in Figure 10. This knot has also been called the Four-Knot (as it is the only 4 crossing knot) and Listing's Knot [7, p 4].

$$
\left(T_{4_{1}}\right)_{x}^{y} \triangleq\left(\sigma_{l}^{-1} \sigma_{r}^{-1}\right)_{a c}^{y b} \cdot\left(\sigma_{r}\right)_{d f}^{c e} \cdot \sigma_{x g}^{a d} \cdot\left(\Omega^{-}\right)_{b e} \cdot\left(\mho^{-}\right)^{g f} .
$$

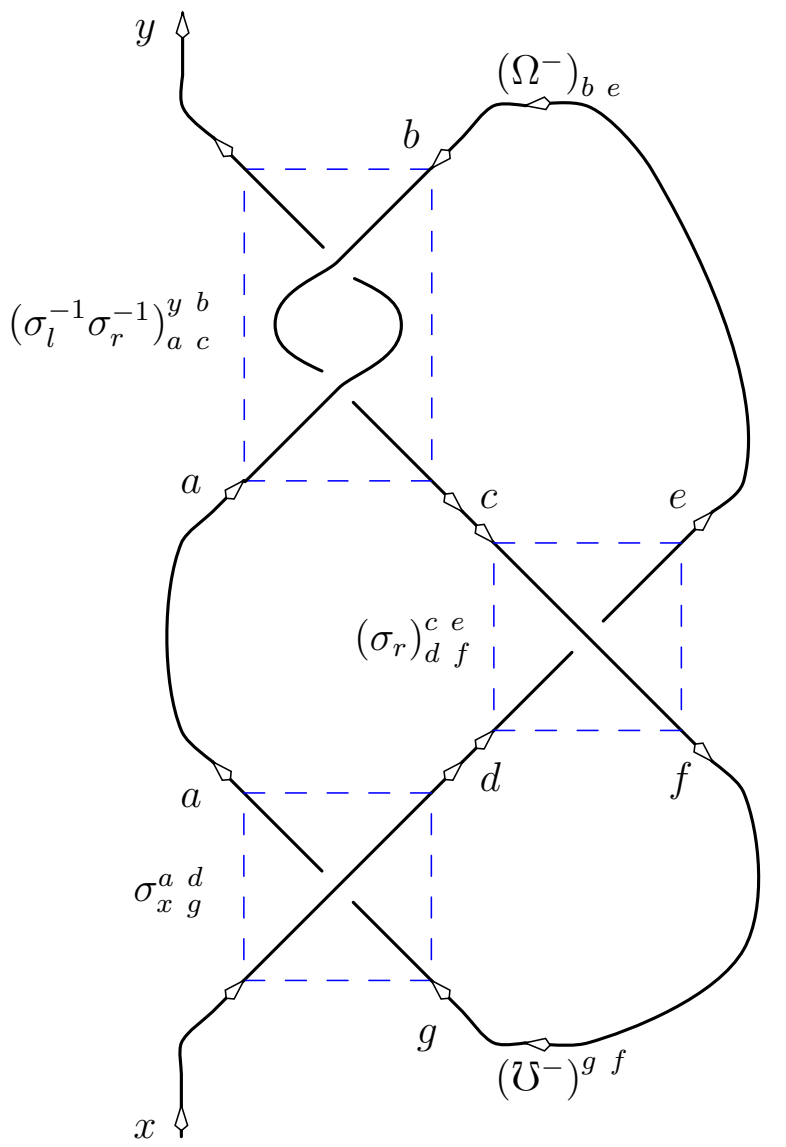

Figure 10: Tangle form of $4_{1}$ (the Figure Eight Knot).

$5_{1}^{2}$ (Whitehead Link): A braid presentation is $\left(\sigma_{1} \sigma_{2}^{-1}\right)^{2} \sigma_{2}^{-1} \in B_{3}$ and a diagram is found in Figure 11. (This link is named after the topologist J H C Whitehead, not the logician Alfred North Whitehead [17, p 200].)

Firstly, we define a temporary tensor to reduce computation:

$$
(W)_{x d}^{c i} \triangleq\left(\sigma^{-2}\right)_{x f}^{c e} \cdot\left(\sigma_{d}^{2}\right)_{h d}^{g i} \cdot\left(\Omega^{+}\right)_{e g} \cdot\left(\mho^{-}\right)^{f h},
$$

where we have written $\sigma^{-2} \triangleq\left(\sigma^{-1}\right)^{2}$. With this, we have:

$$
\left(T_{5_{1}^{2}}\right)_{x}^{y} \triangleq(W)_{x d}^{c i} \cdot\left(\sigma_{r} \sigma_{l}\right)_{i}^{a} b \quad\left(\Omega^{+}\right)_{c a} \cdot\left(\mho^{+}\right)^{d b} .
$$




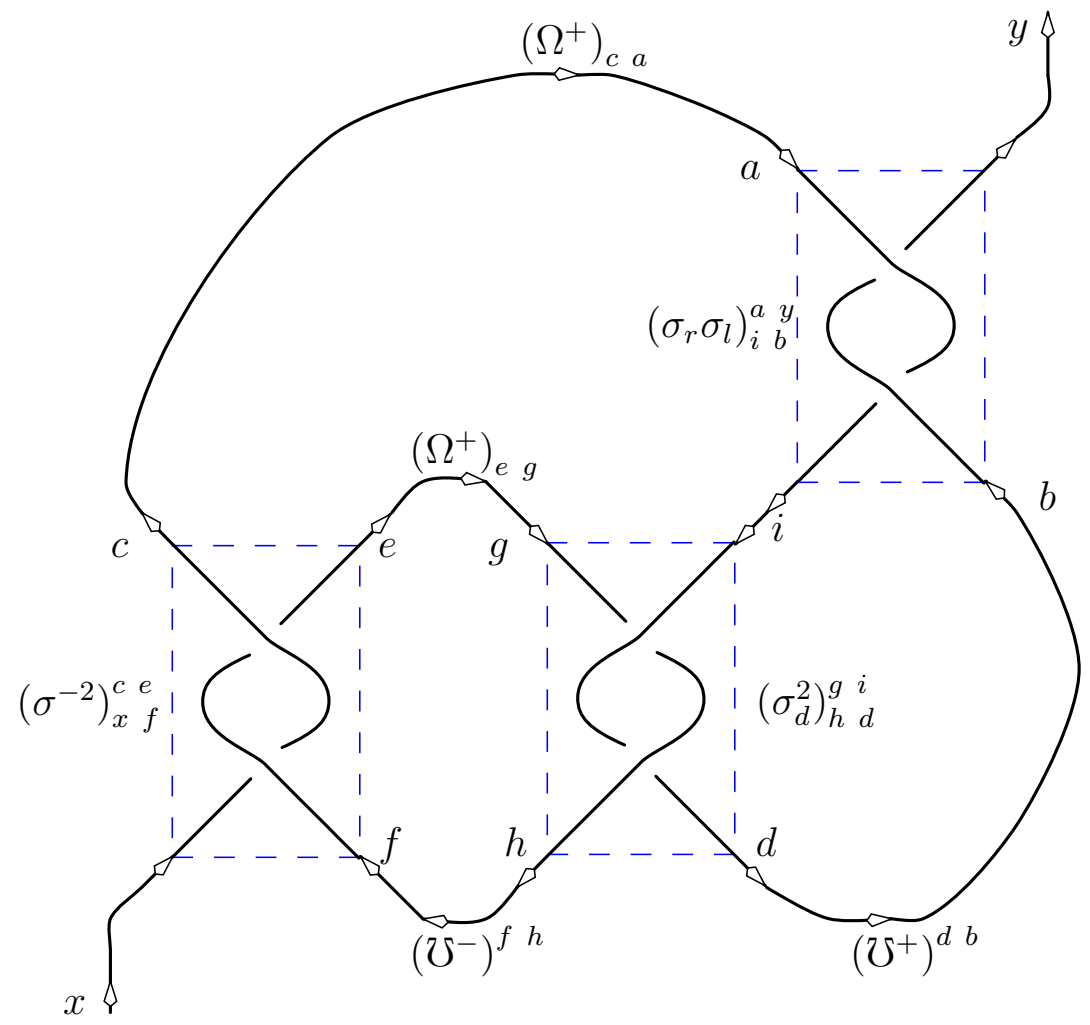

Figure 11: Tangle form of $5_{1}^{2}$ (the Whitehead Link).

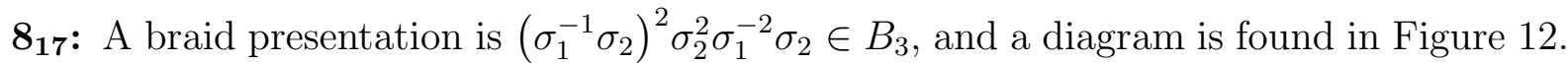
Again, we define some temporary tensors to reduce computation:

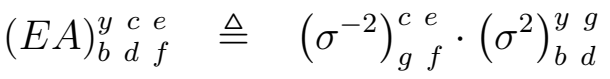

$$
\begin{aligned}
& (E B)_{x i j}^{b{ }_{i} j} \triangleq\left(\sigma^{-1}\right)_{k l}^{d f} \cdot \sigma_{m n}^{b k} \cdot\left(\sigma^{-1}\right)_{o j}^{n l} \cdot \sigma_{x i}^{m o} \text {. }
\end{aligned}
$$

With these, we have:

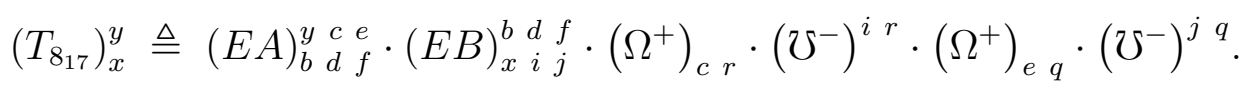

To reduce computation, we may define even more auxiliary tensors:

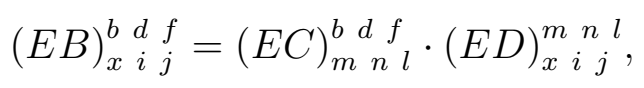

where:

$$
\begin{aligned}
(E C)_{m n^{b l f}}^{b d} & \triangleq\left(\sigma^{-1}\right)_{k l}^{d f} \cdot \sigma_{m n}^{b k} \\
(E D)_{x i j}^{m n^{n} l} & \triangleq\left(\sigma^{-1}\right)_{o j}^{n l}{ }^{n} \cdot \sigma_{x i}^{m o} .
\end{aligned}
$$




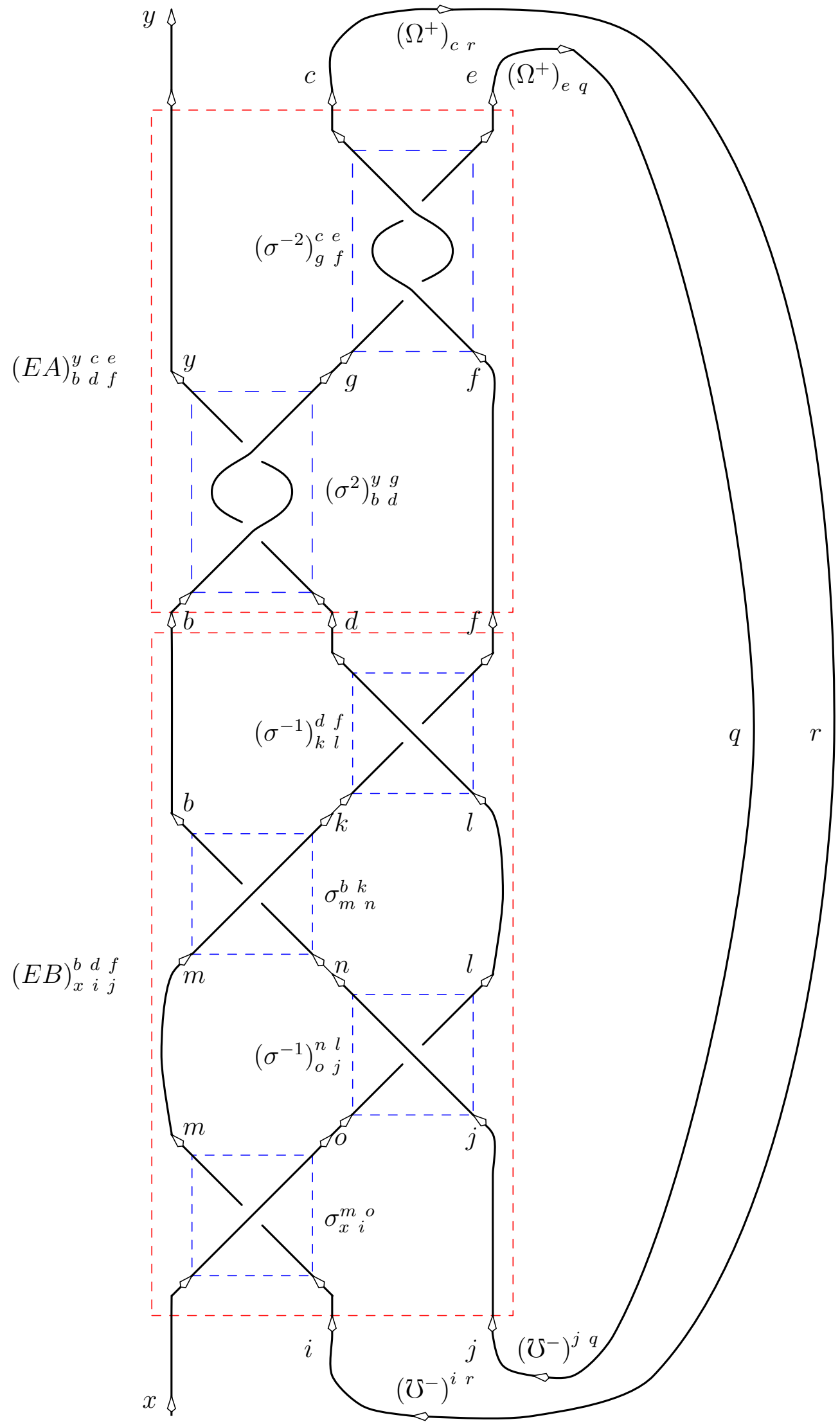

Figure 12: Tangle form of $8_{17}$. 
$\mathbf{9}_{\mathbf{4 2}}$ : A braid presentation is $\sigma_{1}^{3} \sigma_{3} \sigma_{2}^{-1} \sigma_{3} \sigma_{1}^{-2} \sigma_{2}^{-1} \in B_{4}$, and a diagram is found in Figure 14. Again, we define a temporary tensor to reduce computation:

$$
\begin{aligned}
& (N)_{b h}^{a y} \triangleq\left(\sigma_{d}^{2}\right)_{b d}^{a c} \cdot\left(\sigma^{-3}\right)_{f h}^{e y} \cdot\left(\Omega^{-}\right)_{c e} \cdot\left(\mho^{+}\right)^{d f} \cdot \\
& \left(T_{9_{42}}\right)_{x}^{y} \triangleq(N)_{b h}^{a y} \cdot\left(\sigma_{d}^{-1} \sigma^{-1}\right)_{i j}^{b h} \cdot\left(\sigma \sigma_{d}\right)_{x m}^{k i} \cdot\left(\mho^{+}\right)^{m j} \cdot\left(\Omega^{+}\right)_{k a} .
\end{aligned}
$$

10 48: A braid presentation is $\sigma_{1}^{-2} \sigma_{2}^{4} \sigma_{1}^{-3} \sigma_{2} \in B_{3}$, and a diagram is found in Figure 13 .

$$
\begin{aligned}
\left(T_{10_{48}}\right)_{x}^{y} \triangleq & \left(\sigma^{-2}\right)_{b f}^{a y} \cdot\left(\sigma^{4}\right)_{d h}^{f g} \cdot\left(\sigma^{-3}\right)_{c e}^{b d} \cdot(\sigma)_{x i}^{e h} . \\
& \left(\Omega^{-}\right)_{j a} \cdot\left(\mho^{+}\right)^{j c} \cdot\left(\Omega^{+}\right)_{g k} \cdot\left(\mho^{-}\right)^{i k} .
\end{aligned}
$$

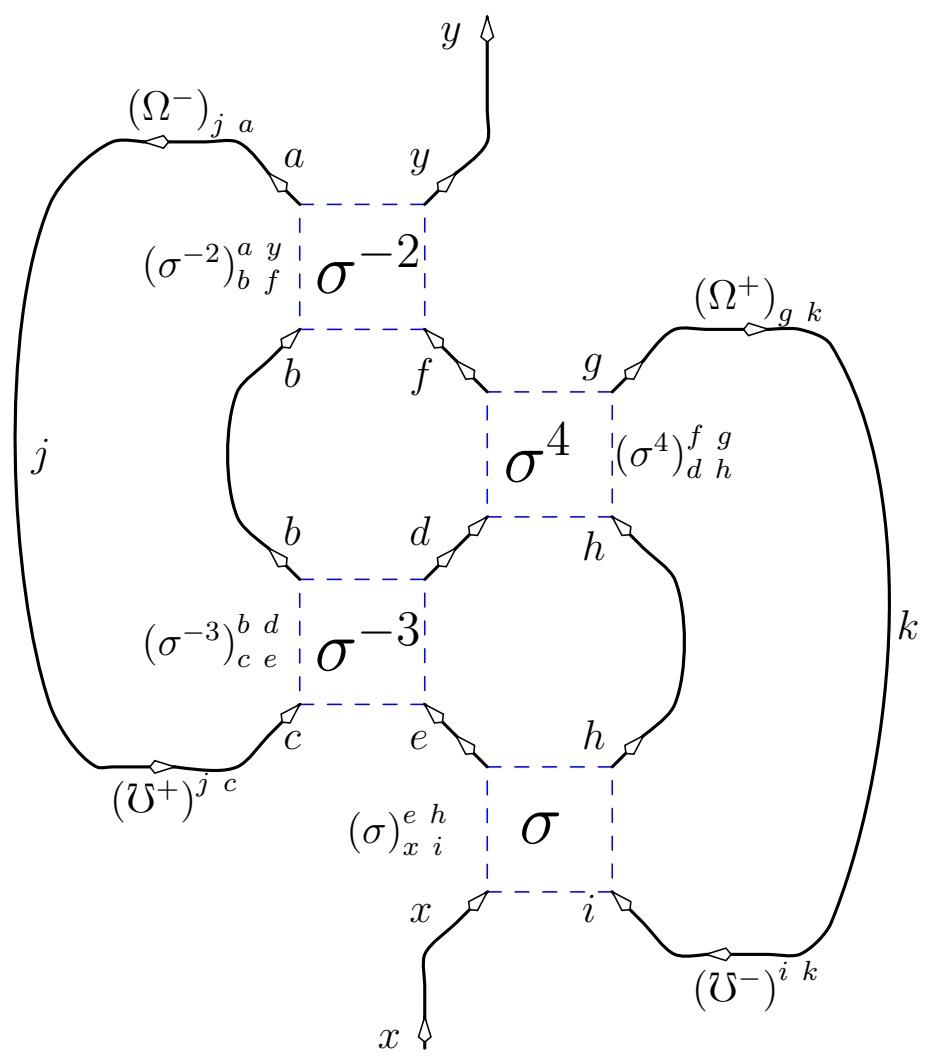

Figure 13: Tangle form of $10_{48}$. 


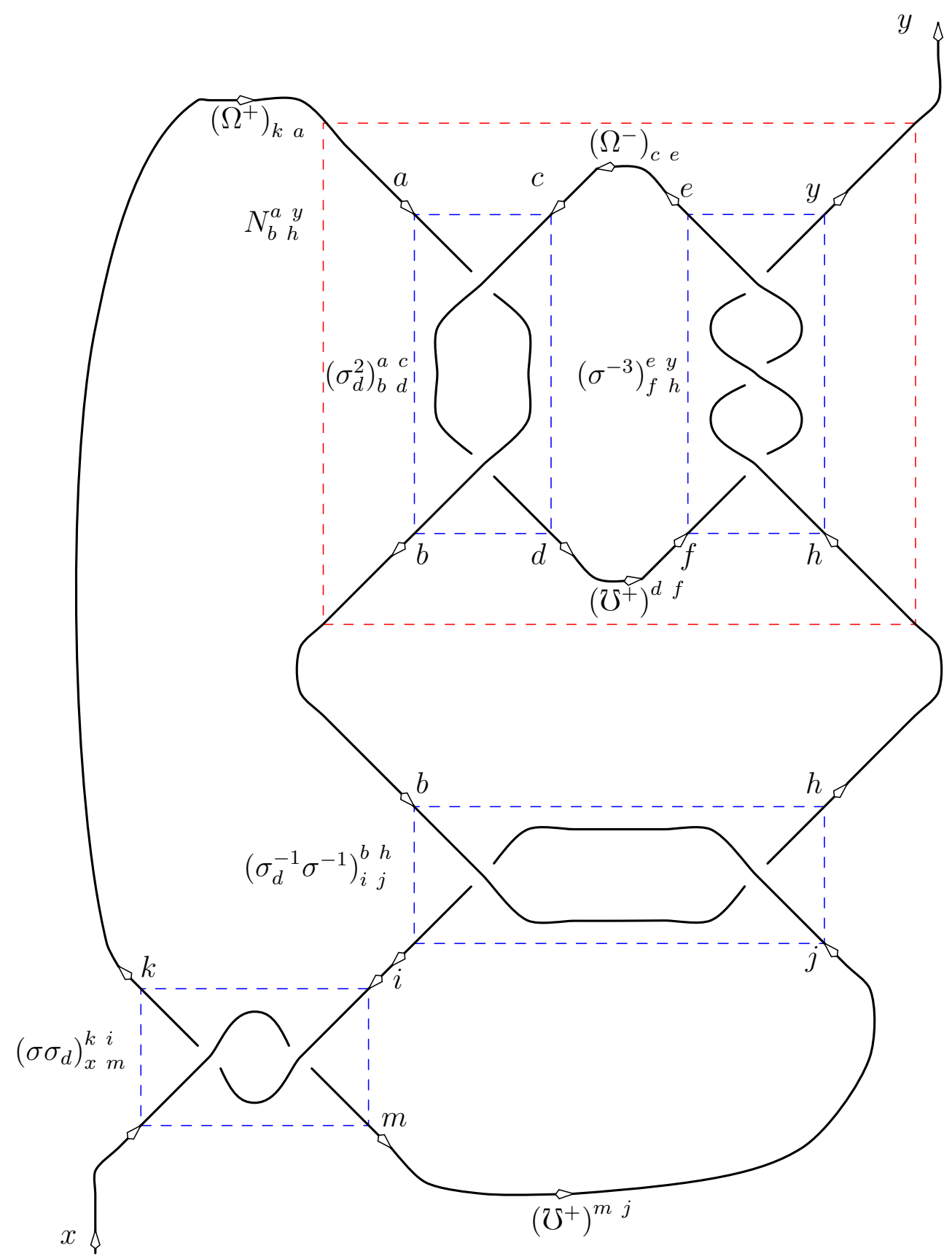

Figure 14: Tangle form of $9_{42}$. 


\section{The Links-Gould Tangle Invariant}

\subsection{Crossing Matrices $\sigma$ and $\sigma^{-1}$}

From the results of $\oint 2$, we have the matrices $\sigma$ and $\sigma^{-1}$, using the substitution $p \triangleq q^{\alpha}$ :

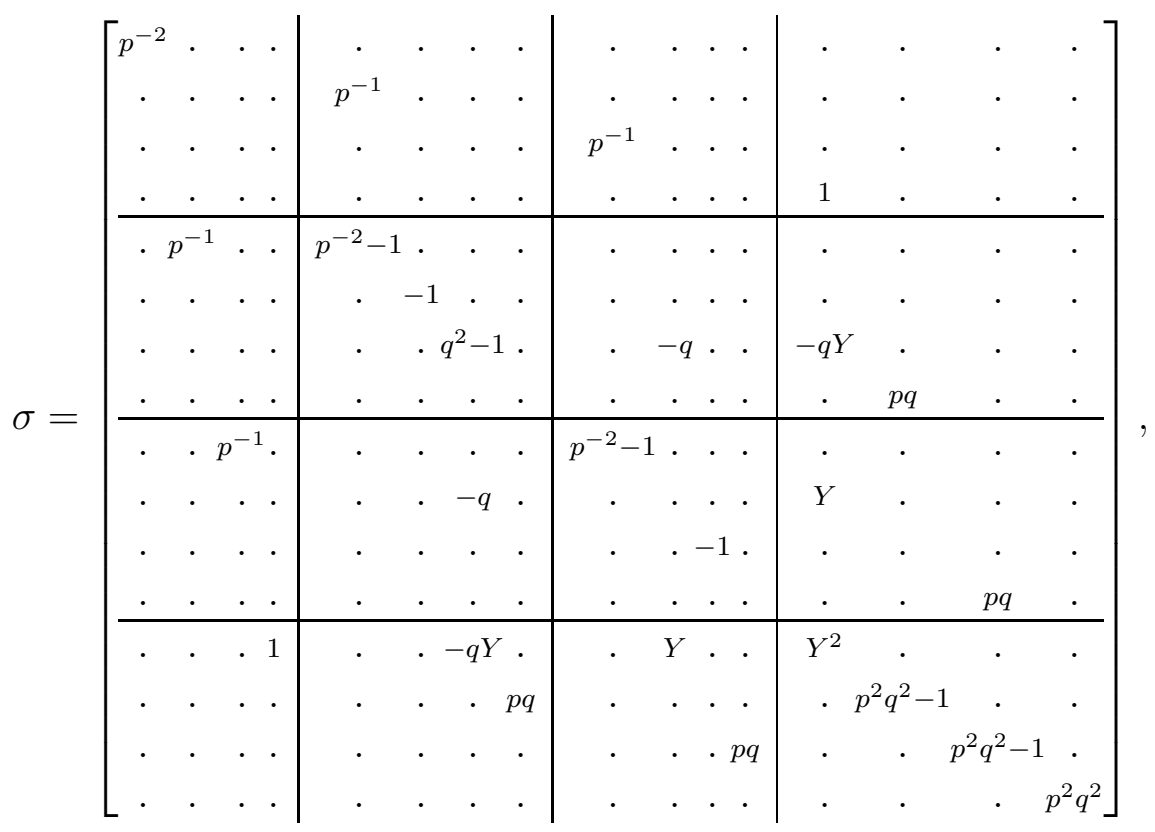

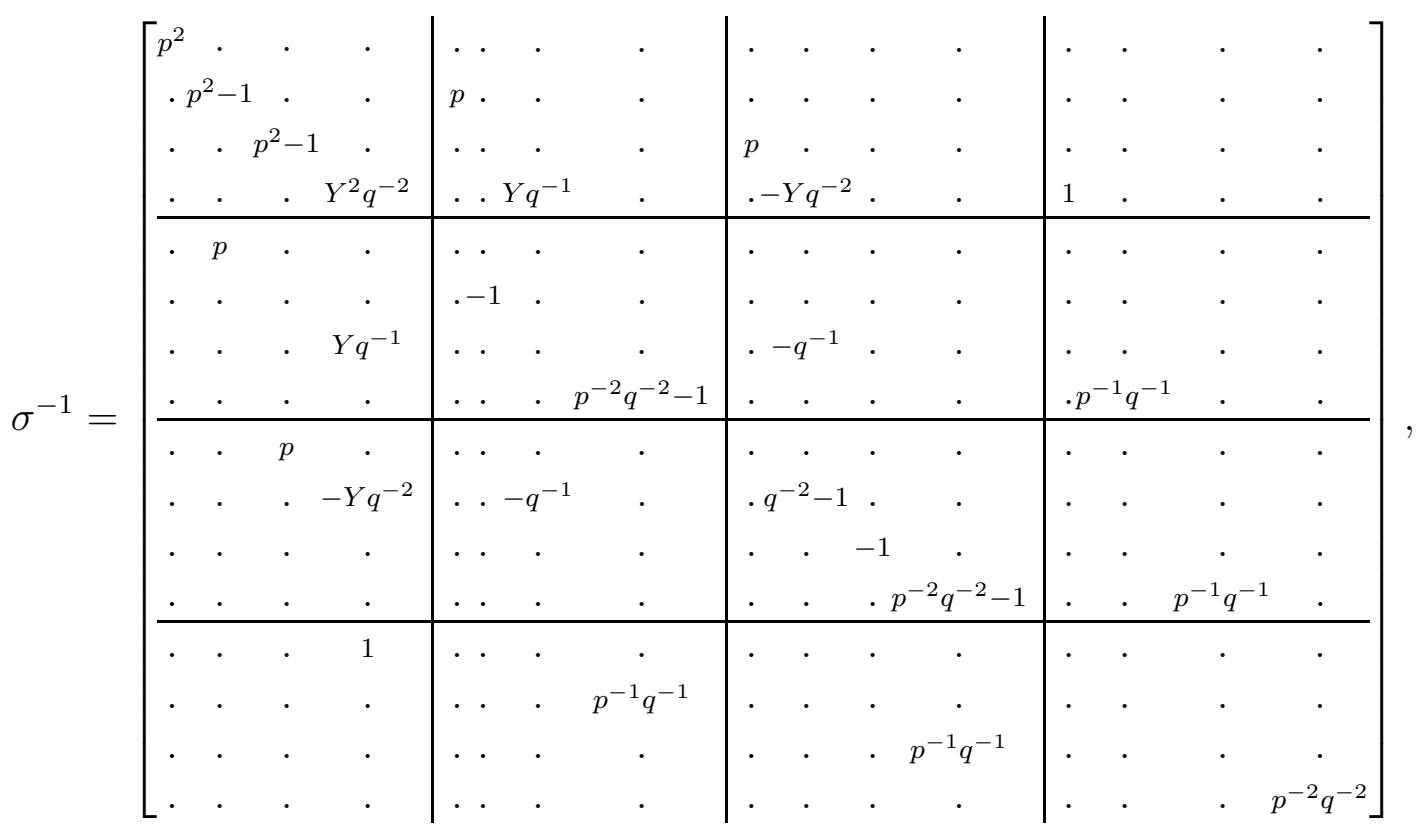

where $Y=\left(p^{-2}-q^{2}+p^{2} q^{2}-1\right)^{1 / 2}$. 


\subsection{Caps and Cups $\Omega^{ \pm}$and $\mho^{ \pm}$}

Where $\mho^{ \pm}=\left(\Omega^{ \pm}\right)^{-1}$, we will use:

$$
\begin{aligned}
& \Omega^{-}=\left[\begin{array}{cccc}
q^{-2 \alpha} & \cdot & \cdot & \cdot \\
\cdot & -q^{-2(\alpha+1)} & \cdot & \cdot \\
\cdot & \cdot & -q^{-2 \alpha} & \cdot \\
\cdot & \cdot & \cdot & q^{-2(\alpha+1)}
\end{array}\right] \equiv\left[\begin{array}{cccc}
p^{-2} & \cdot & \cdot & \cdot \\
\cdot & -p^{-2} q^{-2} & \cdot & \cdot \\
\cdot & \cdot & -p^{-2} & \cdot \\
\cdot & \cdot & \cdot & p^{-2} q^{-2}
\end{array}\right], \\
& \mho^{-}=\left[\begin{array}{cccc}
q^{2 \alpha} & \cdot & \cdot & \cdot \\
\cdot & -q^{2(\alpha+1)} & \cdot & \cdot \\
\cdot & \cdot & -q^{2 \alpha} & \cdot \\
\cdot & \cdot & \cdot & q^{2(\alpha+1)}
\end{array}\right] \equiv\left[\begin{array}{cccc}
p^{2} & \cdot & \cdot & \cdot \\
\cdot & -p^{2} q^{2} & \cdot & \cdot \\
\cdot & \cdot & -p^{2} & \cdot \\
\cdot & \cdot & \cdot & p^{2} q^{2}
\end{array}\right], \\
& \Omega^{+}=\mho^{+}=I_{4} \text {. }
\end{aligned}
$$

The choices for $\Omega^{ \pm}$and $\mho^{ \pm}$are not unique.

- $\Omega^{+}$and $\mho^{+}$may be chosen from consistency considerations in Figure 3. The simple choices:

$$
\left(\Omega^{+}\right)_{a b}=\left(\mho^{+}\right)^{a b}=\delta_{a b}
$$

(i.e. $\Omega^{+}=\mho^{+}=I_{4}$ ), ensure that the definition (8), i.e.

$$
\left(X_{r}\right)_{b d}^{a c} \triangleq X_{f b}^{c g} \cdot\left(\mho^{+}\right)^{a f} \cdot\left(\Omega^{+}\right)_{g d}
$$

(where $X$ is either $\sigma$ or $\sigma^{-1}$ ), simplifies to the elegant form:

$$
\left(X_{r}\right)_{b \quad d}^{a c}=X_{a b}^{c d}
$$

- For the choice of $\Omega^{-}$and $\mho^{-}$, we invoke the following result from [27, Lemma 2, p 354] (see also 26]):

$$
(I \otimes \operatorname{str})\left[\left(I \otimes q^{-2 h_{\rho}}\right) \sigma\right]=k I,
$$

for some constant $k$ depending on the normalisation of $\sigma$. Note that str denotes the supertrace, and that in this case:

$$
\pi\left(q^{-2 h_{\rho}}\right)=\left[\begin{array}{cccc}
q^{-2 \alpha} & \cdot & \cdot & \cdot \\
\cdot & q^{-2 \alpha-2} & \cdot & \cdot \\
\cdot & \cdot & q^{-2 \alpha} & \cdot \\
\cdot & \cdot & \cdot & q^{-2 \alpha-2}
\end{array}\right]
$$


From Figure 8, we require:

$$
\sigma_{x b}^{y a} \cdot\left(\Omega^{+}\right)_{a c} \cdot\left(\mho^{-}\right)^{b c}=\delta_{x}^{y}
$$

which, along with the condition:

$$
\left(\Omega^{-}\right)_{a b} \cdot\left(\mho^{-}\right)^{b c}=\delta_{a}^{c}
$$

imposes the choice:

$$
\begin{aligned}
& \left(\mho^{-}\right)^{b c}=(-)^{[b]} \pi\left(q^{-2 h_{\rho}}\right)_{c}^{b} \\
& \left(\Omega^{-}\right)_{b c}=(-)^{[b]} \pi\left(q^{2 h_{\rho}}\right)_{c}^{b} .
\end{aligned}
$$

For other references on the construction of the cap and cup matrices, see the papers by Reshetikhin and Turaev [35], and particularly Zhang [40] for the superalgebra case.

\subsection{Results}

Some evaluations of the invariant are presented in Table 2. This $U_{q}[g l(2 \mid 1)]$ oriented invariant is an invariant of ambient isotopy.

\begin{tabular}{||l|l||}
\hline \hline$K$ & \multicolumn{1}{|c||}{$L G_{K}(q, p)$} \\
\hline \hline $0_{1}$ & 1 \\
\hline $2_{1}^{2}$ & $-1+p^{-2}-q^{2}+p^{2} q^{2}$ \\
\hline $3_{1}$ & $1+p^{-4}-p^{-2}+2 q^{2}-p^{-2} q^{2}-p^{2} q^{2}-p^{2} q^{4}+p^{4} q^{4}$ \\
\hline $4_{1}$ & $7+\left(p^{-4} q^{-2}+p^{4} q^{2}\right)-3\left(p^{-2}+p^{2}\right)-3\left(p^{-2} q^{-2}+p^{2} q^{2}\right)+2\left(q^{-2}+q^{2}\right)$ \\
\hline $5_{1}^{2}$ & $-10+p^{-6} q^{-2}-3 p^{-4}-3 p^{-4} q^{-2}+4 p^{-2} q^{-2}+9 p^{-2}-2 q^{-2}$ \\
\hline \hline $8_{17}$ & see $\S 4.6$ \\
\hline $9_{42}, 10_{48}$ & see $\S 4.5$ \\
\hline \hline
\end{tabular}

Table 2: The Links-Gould $U_{q}[g l(2 \mid 1)]$ oriented polynomial invariant $L G_{K}(q, p)$, evaluated using the open diagram form of various links $K$. 


\subsection{Behaviour of the Invariant}

Fix a knot $K$, and denote by $K^{*}$ the reflection of $K$ and by $K^{-1}$ the inverse of $K$. From the polynomial for $K$, we may immediately write down the polynomials for $K^{*}$ and $K^{-1}$. For the reflection, we have:

$$
L G_{K^{*}}(q, p)=L G_{K}\left(q^{-1}, p^{-1}\right)
$$

For the inverse, we have:

$$
L G_{K^{-1}}(q, p)=L G_{K}\left(q, q^{-1} p^{-1}\right) .
$$

(this follows from $\alpha \mapsto-(\alpha+1))$.

Chirality: As we have:

$$
K=K^{*} \quad \rightarrow \quad L G_{K}(q, p)=L G_{K^{*}}(q, p),
$$

then we have, conversely, that:

$$
L G_{K}(q, p) \neq L G_{K^{*}}(q, p) \rightarrow K \neq K^{*},
$$

i.e. if the polynomials corresponding to $K$ and $K^{*}$ are distinct, then $K$ must be chiral. Using the identity (9), the test of (10) becomes:

$$
L G_{K}(q, p) \neq L G_{K}\left(q^{-1}, p^{-1}\right) \quad \rightarrow \quad K \neq K^{*}
$$

i.e. if $L G_{K}(q, p)$ is not palindromic, then $K$ is chiral.

Invertibility: We make the observation that the representation of $U_{q}[g l(2 \mid 1)]$ acting on the dual module $V^{*}$ is given by the replacement $\alpha \mapsto-(\alpha+1)$ (with an appropriate redefinition of the Cartan elements). Thus for a given $(1,1)$ tangle $K$, with invariant $L G_{K}(q, p)$, the tangle invariant $L G_{K^{-1}}$ of its inverse $K^{-1}$ is obtained as $L G_{K^{-1}}(q, p)=L G_{K}\left(q, q^{-1} p^{-1}\right)$. However, in view of Proposition 3.2, such an invariant is unable to detect inversion.

We summarise these results in a proposition:

\section{Proposition 4.1}

Let $L G_{K}(q, p)$ be the Links-Gould polynomial invariant for the knot $K$.

- If $L G_{K}(q, p)$ is not invariant under the transformation $q \mapsto q^{-1}$ (which implies $\left.p \mapsto p^{-1}\right)$, then $L G$ detects the chirality of $K$.

- $L G_{K}(q, p)$ enjoys the symmetry property:

$$
L G_{K}(q, p)=L G_{K}\left(q, q^{-1} p^{-1}\right) .
$$




\subsection{The Chirality of $9_{42}$ and $10_{48}$}

The polynomials for $9_{42}$ and $10_{48}$ are:

$$
\begin{aligned}
& L G_{9_{42}}(q, p)= \\
& \quad 3+p^{-8} q^{-6}-2 p^{-6} q^{-6}-2 p^{-6} q^{-4}+p^{-4} q^{-6}+3 p^{-4} q^{-4}+p^{-4} q^{-2}+p^{-4}-p^{-2} q^{-4} \\
& \quad-p^{-2} q^{-2}-3 p^{-2}-3 p^{-2} q^{2}+6 q^{2}+2 q^{4}-p^{2} q^{-2}-p^{2}-3 p^{2} q^{2}-3 p^{2} q^{4}+p^{4} q^{-2} \\
& \quad+3 p^{4}+p^{4} q^{2}+p^{4} q^{4}-2 p^{6}-2 p^{6} q^{2}+p^{8} q^{2} \\
& L G_{10_{48}}(q, p)= \\
& \quad 165+5 p^{-8}-25 p^{-6}+68 p^{-4}-129 p^{-2}-132 p^{2}+67 p^{4}-22 p^{6}+4 p^{8}+p^{-16} q^{-8} \\
& \quad-3 p^{-14} q^{-8}+4 p^{-12} q^{-8}-4 p^{-10} q^{-8}+4 p^{-8} q^{-8}-2 p^{-6} q^{-8}-3 p^{-14} q^{-6}+12 p^{-12} q^{-6} \\
& \quad-21 p^{-10} q^{-6}+24 p^{-8} q^{-6}-22 p^{-6} q^{-6}+13 p^{-4} q^{-6}-3 p^{-2} q^{-6}+16 q^{-4}+5 p^{-12} q^{-4} \\
& \quad-23 p^{-10} q^{-4}+50 p^{-8} q^{-4}-69 p^{-6} q^{-4}+67 p^{-4} q^{-4}-43 p^{-2} q^{-4}-3 p^{2} q^{-4}+94 q^{-2} \\
& \quad-6 p^{-10} q^{-2}+29 p^{-8} q^{-2}-72 p^{-6} q^{-2}+119 p^{-4} q^{-2}-132 p^{-2} q^{-2}-43 p^{2} q^{-2}+13 p^{4} q^{-2} \\
& \quad-2 p^{6} q^{-2}+88 q^{2}-2 p^{-6} q^{2}+12 p^{-4} q^{2}-39 p^{-2} q^{2}-129 p^{2} q^{2}+119 p^{4} q^{2}-69 p^{6} q^{2} \\
& \quad+24 p^{8} q^{2}-4 p^{10} q^{2}+12 q^{4}-2 p^{-2} q^{4}-39 p^{2} q^{4}+68 p^{4} q^{4}-72 p^{6} q^{4}+50 p^{8} q^{4}-21 p^{10} q^{4} \\
& \quad+4 p^{12} q^{4}-2 p^{2} q^{6}+12 p^{4} q^{6}-25 p^{6} q^{6}+29 p^{8} q^{6}-23 p^{10} q^{6}+12 p^{12} q^{6}-3 p^{14} q^{6}-2 p^{6} q^{8} \\
& \quad+5 p^{8} q^{8}-6 p^{10} q^{8}+5 p^{12} q^{8}-3 p^{14} q^{8}+p^{16} q^{8} .
\end{aligned}
$$

Neither of these polynomials are palindromic, hence $L G$ does distinguish the chirality of these knots.

\subsection{The Noninvertibility of $8_{17}$ is not Detected}

Recall that $8_{17}$ is the smallest noninvertible knot. We find its polynomial invariant to be given by:

$$
\begin{aligned}
& L G_{8_{17}}(q, p)= \\
& \quad 139+\left(p^{-12} q^{-6}+p^{12} q^{6}\right)-4\left(p^{-10} q^{-6}+p^{10} q^{6}\right)-4\left(p^{-10} q^{-4}+p^{10} q^{4}\right) \\
& \quad+7\left(p^{-8} q^{-6}+p^{8} q^{6}\right)+18\left(p^{-8} q^{-4}+p^{8} q^{4}\right)+7\left(p^{-8} q^{-2}+p^{8} q^{2}\right)-7\left(p^{-6} q^{-6}+p^{6} q^{6}\right) \\
& \quad-36\left(p^{-6} q^{-4}+p^{6} q^{4}\right)-36\left(p^{-6} q^{-2}+p^{6} q^{2}\right)-7\left(p^{-6}+p^{6}\right)+3\left(p^{-4} q^{-6}+p^{4} q^{6}\right) \\
& \quad+40\left(p^{-4} q^{-4}+p^{4} q^{4}\right)+82\left(p^{-4} q^{-2}+p^{4} q^{2}\right)+40\left(p^{-4}+p^{4}\right)+3\left(p^{-4} q^{2}+p^{4} q^{-2}\right) \\
& \quad-22\left(p^{-2} q^{-4}+p^{2} q^{4}\right)-102\left(p^{-2} q^{-2}+p^{2} q^{2}\right)-102\left(p^{-2}+p^{2}\right)-22\left(p^{-2} q^{2}+p^{2} q^{-2}\right) \\
& \quad+4\left(q^{-4}+q^{4}\right)+68\left(q^{-2}+q^{2}\right) .
\end{aligned}
$$

As $8_{17}$ is amphichiral, the polynomial invariant is palindromic, as predicted by Proposition 4.1. Furthermore, we may observe the invariance:

$$
L G_{8_{17}}(q, p)=L G_{8_{17}}\left(q, q^{-1} p^{-1}\right)
$$

which is consistent with our assertion that our polynomial invariant cannot detect the noninvertibility of any knot. More experiments to illustrate this claim are supplied in $\$ 4.7$. 


\subsection{A Class of Noninvertible Pretzel Knots}

A class of noninvertible knots has been presented by Trotter [38]; this class provides an easily-programmable set of examples to see if a knot invariant detects noninvertibility. Trotter is of the opinion that the knots are chiral. These pretzel knots were in fact the first noninvertible knots to be described [28, p 25].

The structure of the knots $(p, q, r)$ in this family is depicted by its simplest example in Figure 16. Note that $p, q$, and $r$ must all be distinct, odd, and greater than 1. In Figure 16, the notation $X_{r l r}^{N}$ refers to the 2-braid of $N$ crossings formed by the placing of $X_{r}$ atop $X_{l}$, with $X_{r}$ as the top and bottom crossings, for $X$ being either $\sigma$ or $\sigma^{-1}$. The recursive definition for such knots is provided in Figure 15.

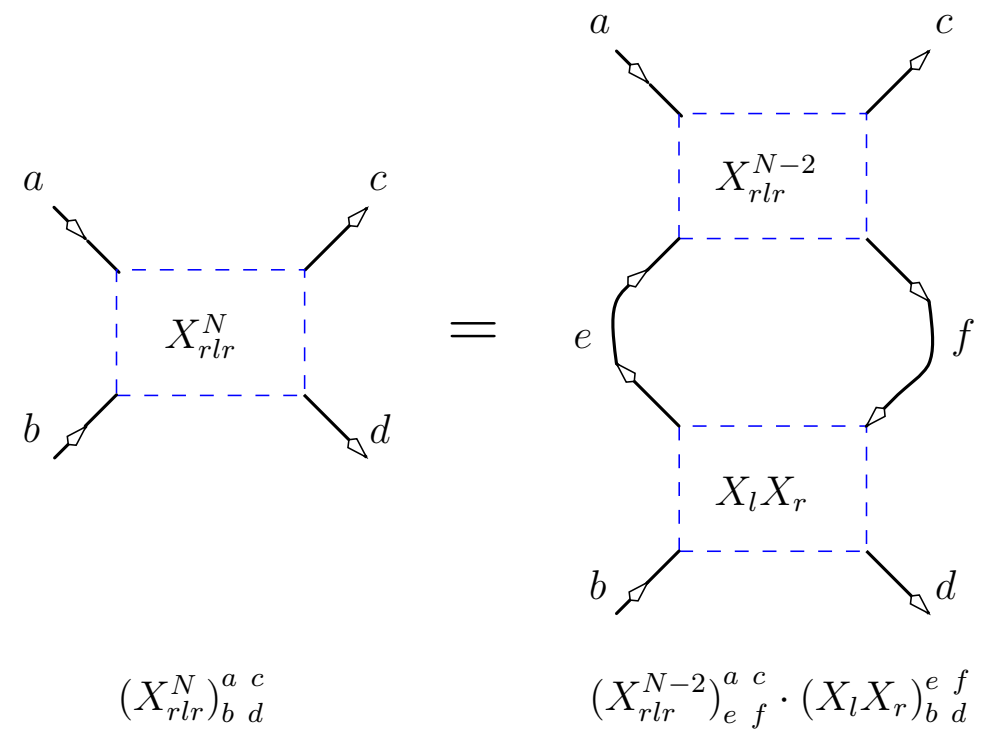

Figure 15: Recursive definition of the towers $X_{r l r}$ used in the evaluation of the LinksGould link invariant for the Trotter pretzel knots; $X$ is either $\sigma$ or $\sigma^{-1}$. The minimum is the case $N=1$, which corresponds to $X_{r}$, i.e. $X_{r l r}^{1} \triangleq X_{r}$. A parallel definition of $X_{l r l}$ might be given.

The tensor associated with the pretzel is:

$$
\begin{aligned}
T_{T P(p, q, r)_{x}}^{y} \triangleq \quad\left(\sigma_{r l r}^{-p}\right)_{x d}^{a c} \cdot\left(\sigma_{r l r}^{-q}\right)_{f h}^{e g} \cdot\left(\sigma_{r l r}^{-r}\right)_{j l}^{i k} & \\
& \left(\Omega^{-}\right)_{a k} \cdot\left(\Omega^{+}\right)_{c e} \cdot\left(\Omega^{+}\right)_{g i} \cdot\left(\mho^{+}\right)^{d f} \cdot\left(\mho^{+}\right)^{h j} \cdot\left(\mho^{+}\right)^{l y}
\end{aligned}
$$

Experiments show that the Links-Gould invariant for this class of noninvertible knots always displays the symmetry of (11), for all $p, q, r \leqslant 67$. This amounts to 5456 knots, the smallest being the $(3,5,7)$ pretzel, a knot of $3+5+7=15$ crossings, and the largest being the $(63,65,67)$ pretzel, a knot of $63+65+67=195$ crossings. Incidentally, we find that the invariant demonstrates that all those pretzels are chiral. 


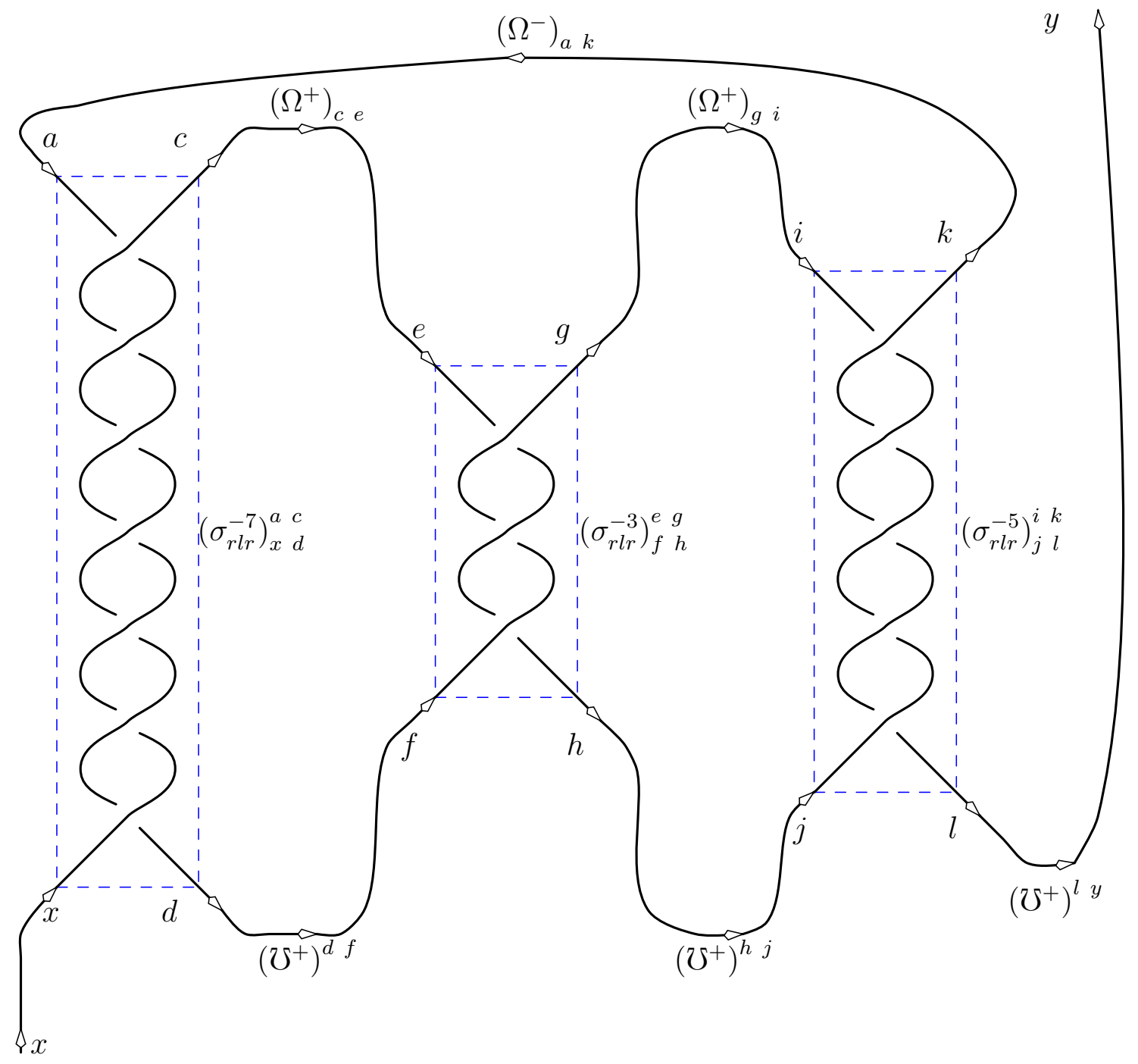

Figure 16: The (noninvertible) pretzel knots of Trotter, in tangle form. This illustration is of the smallest possible one, with $p=7, q=3, r=5$. 


\subsection{The Kinoshita-Terasaka Pair of Mutant Knots}

The Kinoshita-Terasaka pair is an example of a pair of 11 crossing mutant knots that are known to be distinct. To be precise, more commonly, the first of the pair is usually known as the "Kinoshita-Terasaka Knot", and the second has been called the "Conway Knot". In the original source by Kinoshita and Terasaka [21, p 151], the knot involved is the one labelled $\kappa(2,2)$ (reproduced in [28, p 53]). They had constructed this knot as an example of a nontrivial 11 crossing knot with Alexander polynomial equal to 1 . The source used to draw our example is from [1, p 174]; note that these diagrams have 12 crossings, so they are not minimal.

A number of proofs of their distinctness are at hand:

- Adams [1, p 106] states that Francis Bonahon and Lawrence Siebenmann first showed this in 1981. Adams [1, p 174] goes on to state that in 1986 David Gabai [11] showed that their minimal genus Seifert surfaces have different genera[.

- More recently, Morton and Cromwell [29] have constructed a Vassiliev invariant of typef 11 which distinguishes them. This Vassiliev invariant is based on the HOMFLY polynomial for framed links, and the authors compare it with another invariant, itself coming from $S U_{q}(3)$, which does not distinguish them.

More specifically, they show that the ' $S U_{q}(N)$ invariant' for the module with Young diagram $\square$ will distinguish at least some mutant pairs (in particular the KT pair), for all $N \geqslant 4$, but will definitely not distinguish any for $N=2,3$.

More generally, it is known that neither the HOMFLY nor the Kauffman polynomial can distinguish any pair of mutants [1, p 174]. In fact Lickorish [24] used skein theoretical arguments to show this; and furthermore, Lickorish and Lipson [25] and, independently Przytycki [31] again used skein theoretical arguments to show that two equally twisted 2-cables (definition in [1, p 118]) of a mutant pair would have the same HOMFLY polynomial. Perhaps the strongest statement that can be made in this direction was provided in 1994 by Chmutov, Duzhin and Lando [5], who proved that all Vassiliev invariants of type less than 9 will agree on any pair of mutants.

The question of whether the Links-Gould invariant is able to distinguish mutants is immediately answerable in the negative. Theorem 5 of [29] states that if the modules occurring in the decomposition of $V \otimes V$ each have unit multiplicity, as indeed (1) shows in our case, then the invariant is unable to detect mutations. Whilst this was proved in 229 for the case of quantum algebras, the extension to the case of quantum superalgebras is quite straightforward. As an example, we have explicitly evaluated the Links-Gould invariant for the aforementioned pair of mutants.

\footnotetext{
${ }^{3}$ If $L$ is an oriented link in $S^{3}$ (i.e. the 3 -sphere), then a Seifert surface for $L$ is an oriented surface $R$ embedded in $S^{3}$ such that $\partial R=L$ and no component is closed. [11, p 677]. That is, a Seifert surface is a 2-manifold with boundary being the link in question; the genus of such a surface being a topological classifying label [1, p 95-106]. The original reference for the Seifert algorithm is contained in [37.

${ }^{4} \mathrm{~A}$ Vassiliev invariant is defined [29, p 229] to be of type $d$ if it is zero on any link diagram of $d+1$ nodes, and to be of degree $d$ if it is of type $d$ but not of type $d-1$.
} 
We illustrate $K T$, the first of Kinoshita-Terasaka pair, in Figure 17, where the tensors $K T A$ and $K T B$ are defined below, in Figures 19 and 18. From $K T$, we may build the mutant $K T^{\prime}$ by replacing the component $K T A$ with $K T A^{\prime}$ (depicted in Figure 20), which is formed by reflection of $K T A$ about a horizontal line.

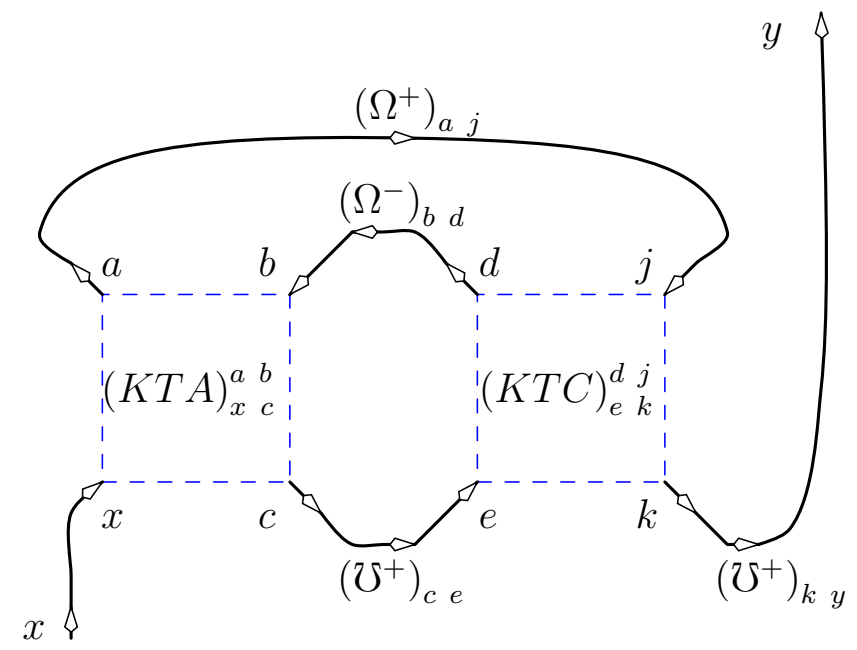

Figure 17: $K T$, the first of the Kinoshita-Terasaka pair of mutant knots, where the subdiagrams $K T A, K T A^{\prime}$ and $K T C$ are found in Figures 19, 20 and 18 respectively. (The mutant $K T^{\prime}$ of $K T$ is obtained by exchanging $K T A$ with $K T A^{\prime}$.)

The tensors associated with $K T$ and $K T^{\prime}$ are:

$$
\begin{aligned}
\left(T_{K T}\right)_{x}^{y} \triangleq(K T A)_{x c}^{a b} \cdot(K T C)_{e k}^{d j} \cdot\left(\Omega^{-}\right)_{b d} \cdot\left(\mho^{+}\right)^{c e} \cdot\left(\Omega^{+}\right)_{a j} \cdot\left(\mho^{+}\right)^{k y} \\
\left(T_{K T^{\prime}}\right)_{x}^{y} \triangleq\left(K T A^{\prime}\right)_{x c}^{a b} \cdot(K T C)_{e k}^{d j} \cdot\left(\Omega^{-}\right)_{b d} \cdot\left(\mho^{+}\right)^{c e} \cdot\left(\Omega^{+}\right)_{a j} \cdot\left(\mho^{+}\right)^{k y},
\end{aligned}
$$

where

$$
\begin{aligned}
(K T A)_{q c}^{a b} & \triangleq\left(\sigma \sigma_{d}\right)_{d e}^{a b} \cdot\left(\sigma^{-2}\right)_{q g}^{d f} \cdot\left(\sigma_{d}^{-1}\right)_{i c}^{h e} \cdot\left(\Omega^{+}\right)_{f h} \cdot\left(\mho^{-}\right)^{g i} \\
\left(K T A^{\prime}\right)_{q c}^{a b} & \triangleq\left(\sigma^{-2}\right)_{d g}^{a j} \cdot\left(\sigma_{d}^{-1}\right)_{i e}^{h b} \cdot\left(\sigma \sigma_{d}\right)_{q c}^{d e} \cdot\left(\Omega^{+}\right)_{f h} \cdot\left(\mho^{-}\right)^{g i} \\
(K T C)_{e k}^{d j} & \triangleq(K T B)_{e g}^{d f} \cdot\left(\sigma_{l}^{-1} \sigma_{r}^{-1}\right)_{i k}^{h j} \cdot\left(\Omega^{-}\right)_{f h} \cdot\left(\mho^{+}\right)^{g i} \\
(K T B)_{e g}^{d f} & \triangleq \sigma_{a c}^{d b} \cdot\left(\sigma_{d}^{2}\right)_{m n}^{l f} \cdot\left(\sigma^{-1} \sigma_{d}^{-1}\right)_{e g}^{a n} \cdot\left(\Omega^{+}\right)_{b l} \cdot\left(\mho^{-}\right)^{c m} .
\end{aligned}
$$




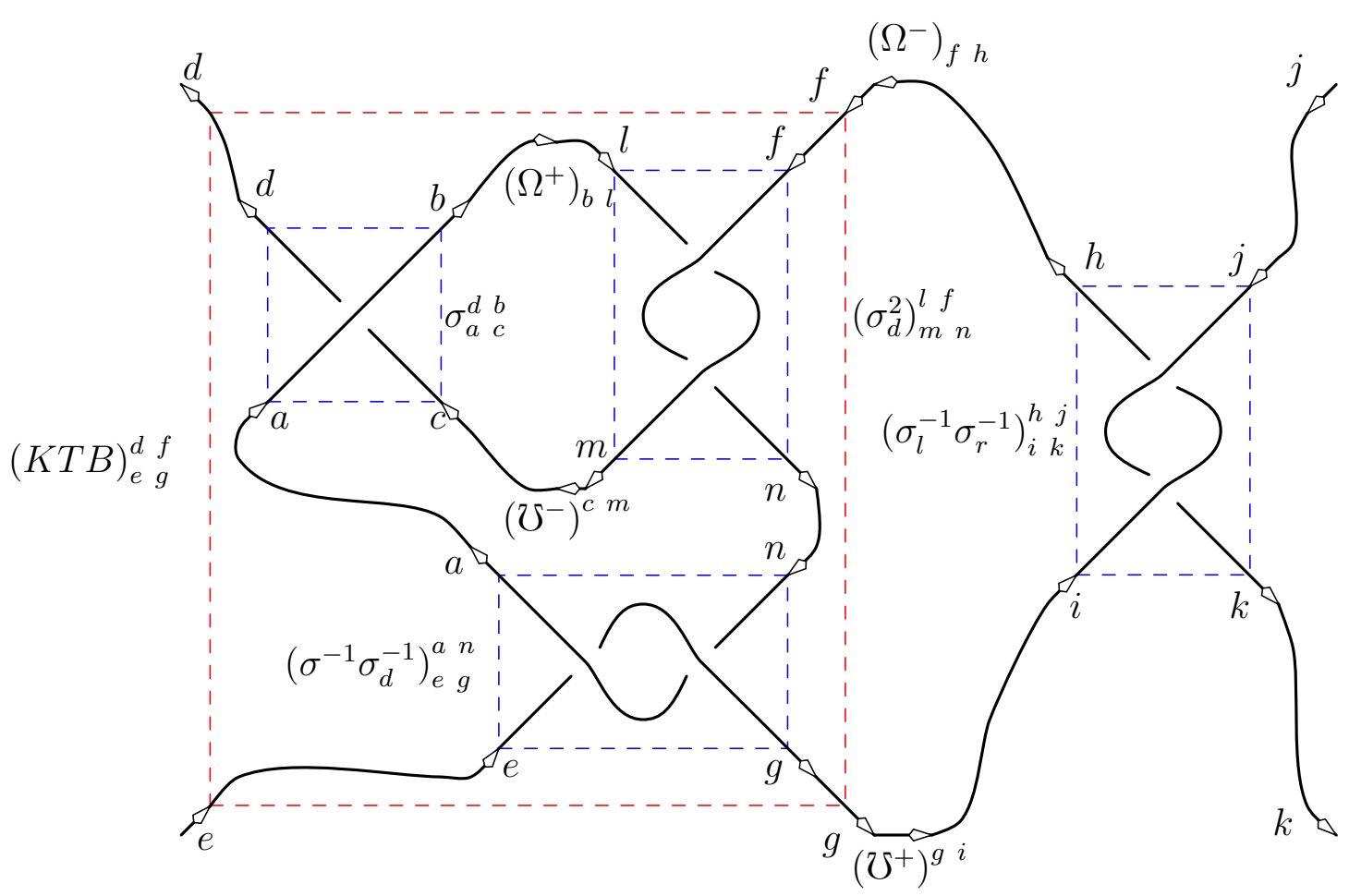

Figure 18: The component $K T C$ of the Kinoshita-Terasaka pair of mutant knots $K T$ and $K T^{\prime}$.

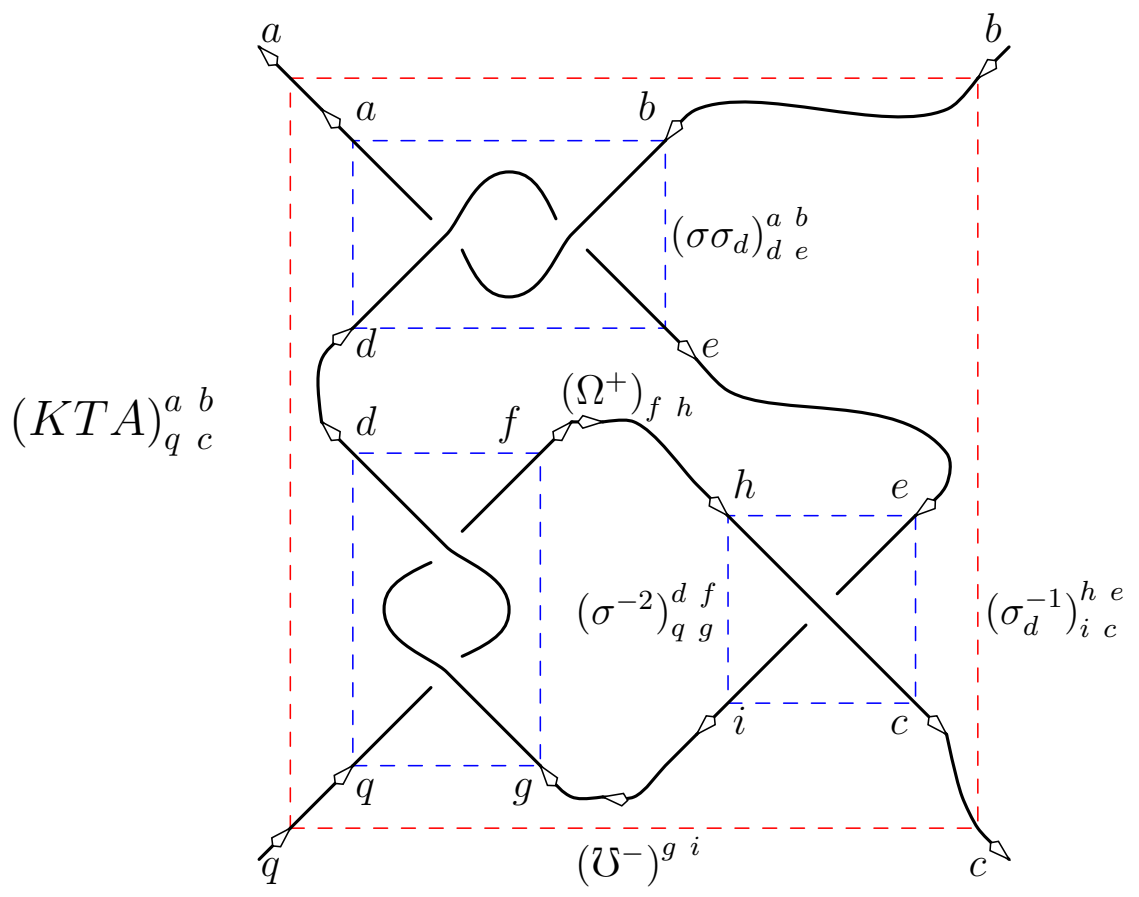

Figure 19: The component KTA of $K T$, the first of the Kinoshita-Terasaka pair. 


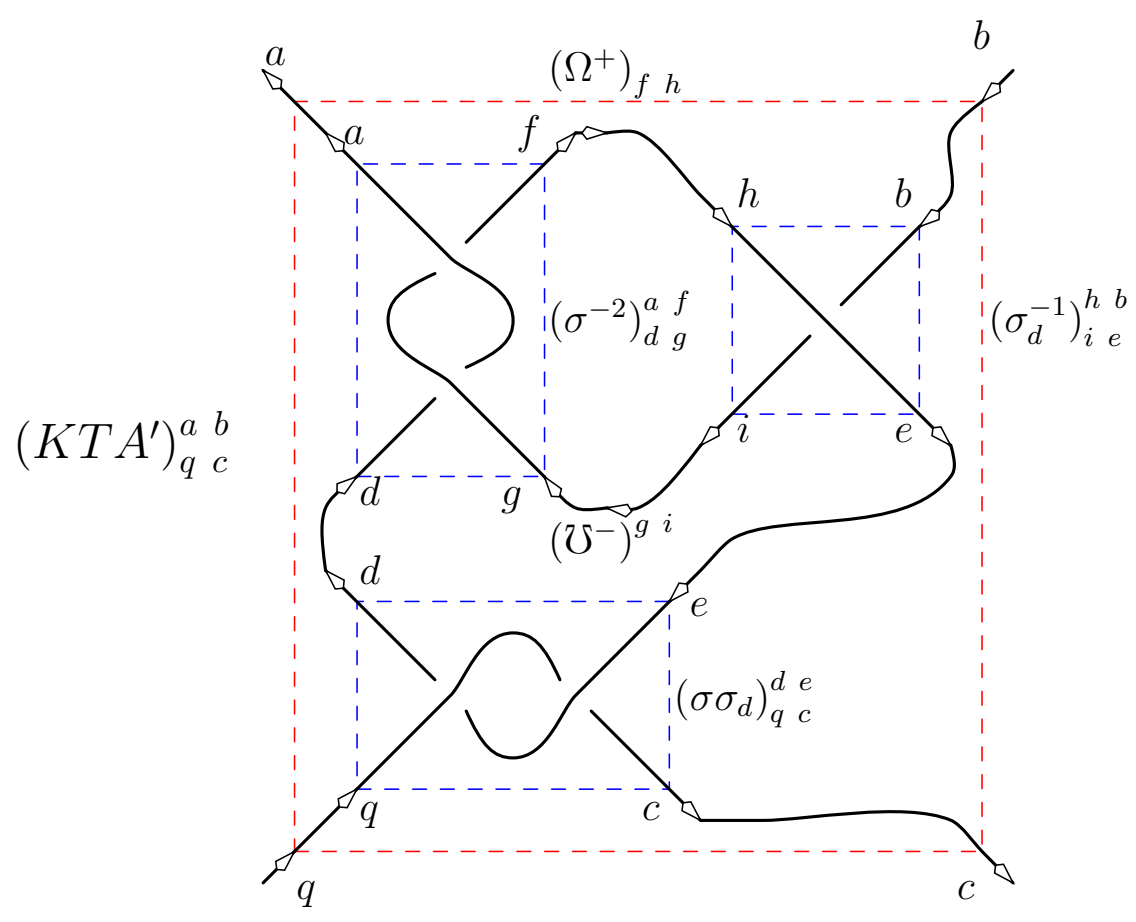

Figure 20: The component $K T A^{\prime}$ of $K T^{\prime}$, the second of the Kinoshita-Terasaka pair.

\subsection{Links-Gould Polynomials of the K-T Mutants}

We find the Links-Gould polynomials of both mutants to be:

$$
\begin{aligned}
& L G_{K T}(q, p)= \\
& \quad-23-p^{-6} q^{-8}-p^{-6} q^{-6}+2 p^{-6} q^{-4}+p^{-6} q^{-2}-p^{-6}+p^{-4} q^{-8}+6 p^{-4} q^{-6}-3 p^{-4} q^{-4} \\
& \quad-9 p^{-4} q^{-2}+2 p^{-4}+3 p^{-4} q^{2}-7 p^{-2} q^{-6}-7 p^{-2} q^{-4}+18 p^{-2} q^{-2}+9 p^{-2}-11 p^{-2} q^{2} \\
& \quad-2 p^{-2} q^{4}+2 q^{-6}+14 q^{-4}-8 q^{-2}+6 q^{2}+10 q^{4}-7 p^{2} q^{-4}-7 p^{2} q^{-2}+18 p^{2}+9 p^{2} q^{2} \\
& \quad-11 p^{2} q^{4}-2 p^{2} q^{6}+p^{4} q^{-4}+6 p^{4} q^{-2}-3 p^{4}-9 p^{4} q^{2}+2 p^{4} q^{4}+3 p^{4} q^{6}-p^{6} q^{-2}-p^{6} \\
& \quad+2 p^{6} q^{2}+p^{6} q^{4}-p^{6} q^{6},
\end{aligned}
$$

hence the Links-Gould link invariant does not distinguish between these mutants.

As predicted by the theorem of [29], the tensors $K T A$ and $K T A^{\prime}$ are in fact identical, which explains why the pair of mutants yield the same invariant. 


\section{Acknowledgements}

Louis Kauffman thanks the National Science Foundation for support of this research under grant number DMS-9205277 and the NSA for partial support under grant number MSPF-96G-179.

Jon Links is supported by an Australian Postdoctoral Research Fellowship and a University of Queensland New Staff Research Grant. The authors wish to thank Mark Gould for his enthusiastic support of this research.

\section{References}

[1] Colin C Adams. The Knot Book: an Elementary Introduction to Mathematical Theory of Knots. Freeman, New York, 1994.

[2] James W Alexander. Topological invariants of knots and links. Transactions of the American Mathematical Society, 20:275-306, 1923.

[3] James W Alexander and G B Briggs. On types of knotted curves. Annals of Mathematics, 28:562-586, 1926-1927.

[4] Dror Bar-Natan. On the Vassiliev knot invariants. Topology, 34(2):423-472, 1995.

[5] S V Chmutov, S V Duzhin, and S K Lando. Vassiliev knot invariants. I. introduction. singularities and bifurcations. Advances in Soviet Mathematics, 21:117-126, 1994.

[6] John H Conway. An enumeration of knots and links, and some of their algebraic properties. In Leech [23], pages 329-358. Proceedings of a Conference in Oxford, 1967.

[7] Richard H Crowell and Ralph H Fox. Introduction to Knot Theory. Number 57 in Graduate Texts in Mathematics. Springer-Verlag, Berlin, New York, Springer edition, 1977.

[8] Helmut Doll and Jim Hoste. A tabulation of oriented links. Mathematics of Computation, 57(196):747-761, 1991.

[9] V G Drinfel'd. Quantum groups. In Andrew M Gleason, editor, Proceedings of the International Congress of Mathematicians 1986 (2 volumes), pages 798-820, Providence, Rhode Island, USA, 1987. American Mathematical Society.

[10] Peter Freyd, David N Yetter, Jim Hoste, W B Raymond Lickorish, Kenneth C Millet, and Adrian Ocneanu. A new polynomial invariant of knots and links. Bulletin of the American Mathematical Society (New Series), 12(2):239-246, April 1985. Included in [22, pp 12-19].

[11] David Gabai. Genera of the alternating links. Duke Mathematical Journal, 53(3):677$681,1986$. 
[12] Mark D Gould, Jon R Links, and Yao-Zhong Zhang. Type-I quantum superalgebras, $q$-supertrace and two-variable link polynomials. Journal of Mathematical Physics, 37:987-1003, 1996.

[13] Michio Jimbo. A $q$-difference analogue of $U(\mathfrak{g})$ and the Yang-Baxter equation. Letters in Mathematical Physics, 10(1):63-69, 1985.

[14] Vaughan F R Jones. A polynomial invariant for knots via Von Neumann algebras. Bulletin of the American Mathematical Society, 12(1):103-111, January 1985. Included in [22, pp 3-11].

[15] Vaughan F R Jones. Hecke algebra representations of braid groups and link polynomials. Annals of Mathematicals, 126:335-388, 1987. Included in [22, pp 20-73].

[16] Louis H Kauffman. On Knots. Number 115 in Annals of Mathematics Studies. Princeton University Press, Princeton, NJ, 1987.

[17] Louis H Kauffman. New invariants in the theory of knots. The American Mathematical Monthly, 95(3):195-242, March 1988.

[18] Louis H Kauffman. Knots and Physics. World Scientific, Singapore, 2nd edition, 1993.

[19] Louis H Kauffman. Knots and diagrams. In Shin'ichi Suzuki, editor, Lectures at Knots96, pages 123-194. World Scientific, 1997.

[20] S M Khoroshkin and V N Tolstoy. Universal $R$-matrix for quantized (super)algebras. Communications in Mathematical Physics, 141(3):599-617, 1991.

[21] Shin'ichi Kinoshita and Hidetaka Terasaka. On unions of knots. Osaka Mathematical Journal, 9:131-153, 1957.

[22] Toshitake Kohno, editor. New Developments in the Theory of Knots, volume 11 of Advanced Series in Mathematical Physics. World Scientific, Singapore, 1990.

[23] John Leech, editor. Computational Problems in Abstract Algebra, Oxford, UK, 1970. Pergamon Press. Proceedings of a Conference in Oxford, 1967.

[24] W B Raymond Lickorish. Linear skein theory and link polynomials. Topology and its Applications, 27(3):265-274, 1987.

[25] W B Raymond Lickorish and Andrew S Lipson. Polynomials of 2-cable-like links. Proceedings of the American Mathematical Society, 100(2):355-361, 1987.

[26] Jon R Links and Mark D Gould. Two variable link polynomials from quantum supergroups. Letters in Mathematical Physics, 26(3):187-198, November 1992.

[27] Jon R Links, Mark D Gould, and Rui Bin Zhang. Quantum supergroups, link polynomials and representation of the braid generator. Reviews in Mathematical Physics, 5(2):345-361, 1993.

[28] Charles Livingston. Knot Theory, volume 24 of The Carus Mathematical Monographs. The Mathematical Association of America, Washington, DC, 1993. 
[29] Hugh R Morton and Peter R Cromwell. Distinguishing mutants by knot polynomials. Journal of Knot Theory and its Ramifications, 5(2):225-238, 1996.

[30] Viktor V Prasolov and A B Sossinsky. Knots, Links, Braids and 3-Manifolds: An Introduction to the New Invariants in Low-Dimensional Topology, volume 154 of Translations of Mathematical Monographs. American Mathematical Society, Providence, Rhode Island, USA, 1996. Translated from the Russian manuscript by Sossinsky.

[31] Józef H Przytycki. Equivalence of cables of mutants of knots. Canadian Journal of Mathematics, 41(2):250-273, 1989.

[32] Józef H Przytycki and Paweł Traczyk. Invariants of links of Conway type. Kobe Journal of Mathematics, 4(2):115-139, 1987.

[33] Kurt Reidemeister. Knotentheorie. Ergebnisse der Mathematik und ihrer Grenzgebiete; Bd 1 Hft 1. Chelsea, New York, 1948. First published in German in 1932.

[34] Nikolai Yu Reshetikhin. Quantized universal enveloping algebras, the Yang-Baxter equation and invariants of links I, II. L.O.M.I. preprints, numbers E-4-87 and E-1787, never published, 1987.

[35] Nikolai Yu Reshetikhin and Vladimir G Turaev. Ribbon graphs and their invariants derived from quantum groups. Communications in Mathematical Physics, 127:1-26, 1990 .

[36] Dale Rolfsen. Knots and Links, volume 7 of Mathematics Lecture Series. Publish or Perish, Inc, Wilmington, Delaware, USA, 1976.

[37] Herbert Seifert. Uber das Geschlecht von Knoten. Mathematische Annalen, 110:571592, August 1934. In German.

[38] Hale F Trotter. Non-invertible knots exist. Topology, 2:275-280, 1964.

[39] Vladimir G Turaev. The Yang-Baxter equation and invariants of links. Inventiones Mathematicae, 92(3):527-553, 1988.

[40] Rui Bin Zhang. Quantum supergroups and topological invariants of three-manifolds. Review of Mathematical Physics, 7(5):809-831, 1995.

[41] Rui Bin Zhang, Mark D Gould, and Anthony J Bracken. Quantum group invariants and link polynomials. Communications in Mathematical Physics, 137(1):13-27, 1991. 